\title{
Zambia: Statistical Appendix
}

This Statistical Appendix for Zambia was prepared by a staff team of the International Monetary Fund as background documentation for the periodic consultation with the member country. It is based on the information available at the time it was completed on November 20, 2007. The views expressed in this document are those of the staff team and do not necessarily reflect the views of the government of Zambia or the Executive Board of the IMF.

The policy of publication of staff reports and other documents by the IMF allows for the deletion of market-sensitive information.

To assist the IMF in evaluating the publication policy, reader comments are invited and may be sent by e-mail to publicationpolicy@imf.org.

Copies of this report are available to the public from

International Monetary Fund $\bullet$ Publication Services

700 19th Street, N.W. • Washington, D.C. 20431

Telephone: (202) $6237430 \bullet$ Telefax: (202) 6237201

E-mail: publications@imf.org • Internet: http://www.imf.org

Price: $\$ 18.00$ a copy

\section{International Monetary Fund Washington, D.C.}





\title{
INTERNATIONAL MONETARY FUND
}

\author{
ZAMBIA \\ Statistical Appendix \\ Prepared by Phebby Kufa (STA); Patrick Akatu and Jan Mikkelsen (both AFR) \\ Approved by the African Department
}

November 20, 2007

Contents

Page

Statistical Appendix Tables

Basic Indicators. 3

1. Gross Domestic Product by Sector of Origin at Constant Prices..........................................

2. Gross Domestic Product by Sector of Origin at Current Prices.............................................6

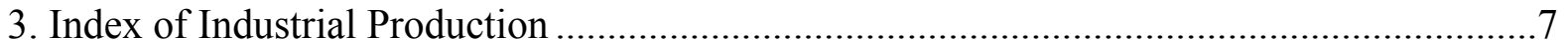

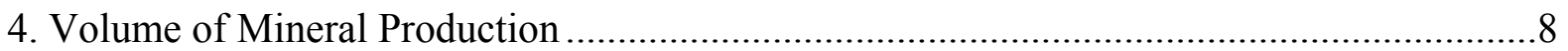

5. Marketed Production of Selected Agricultural Crops.......................................................

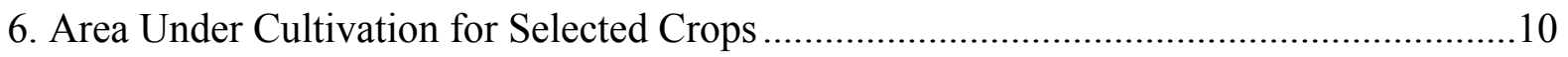

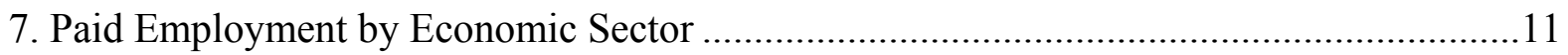

8. Annual Average Composite Index of Consumer Prices ...................................................12

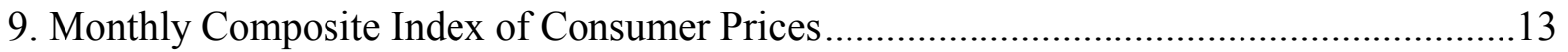

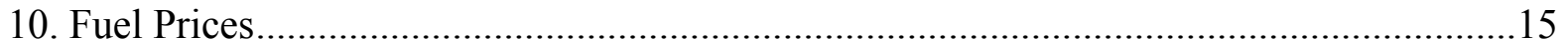

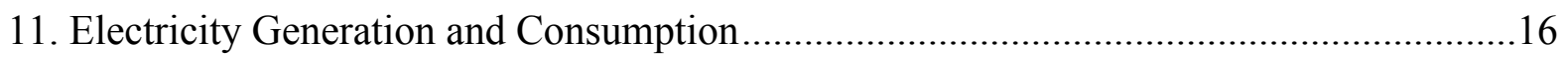

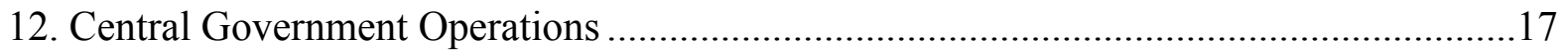

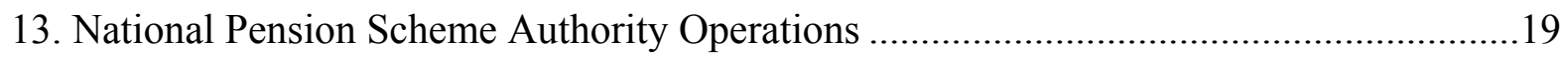

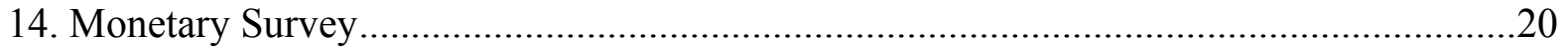

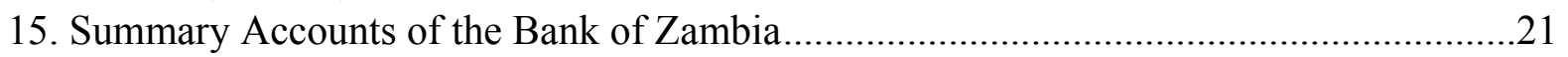

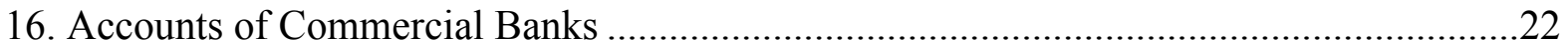

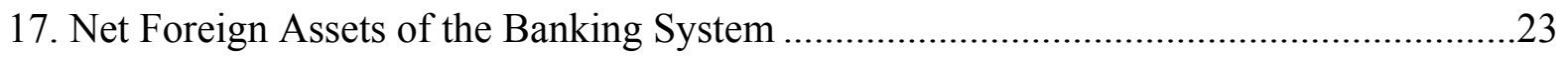

18. Commercial Bank Loans and Advances by Sector ..........................................................24

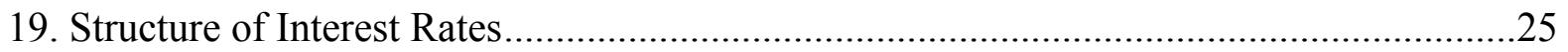

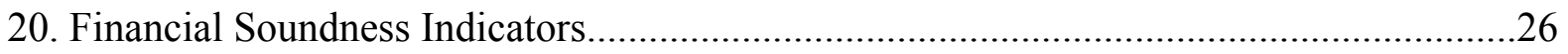

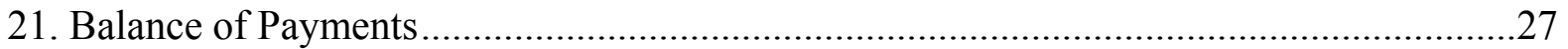

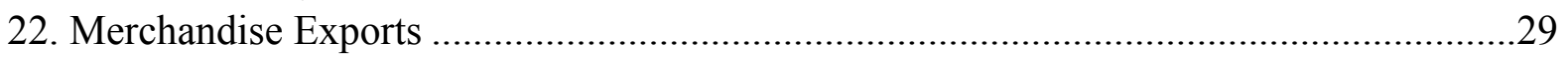

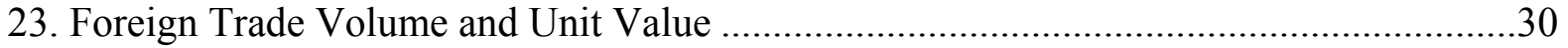

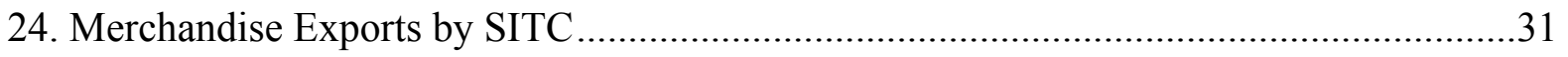

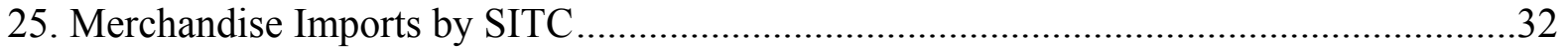

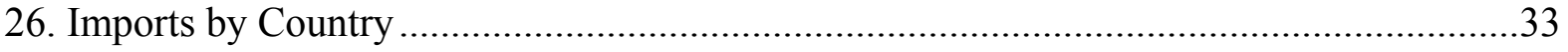




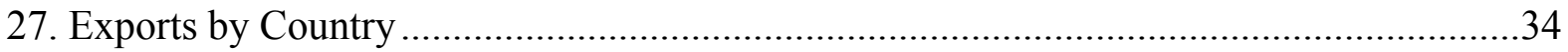

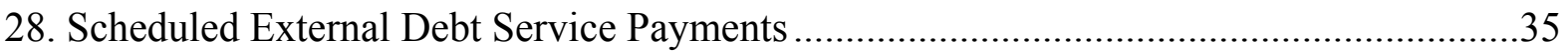

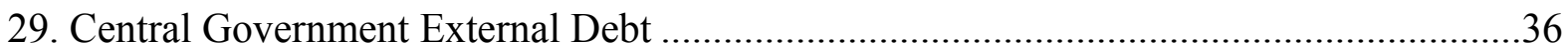

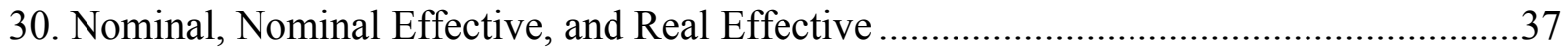

31. Summary of Consolidated Foreign Exchange Market.................................................38

\section{Annexes}

I. Millennium Development Goals

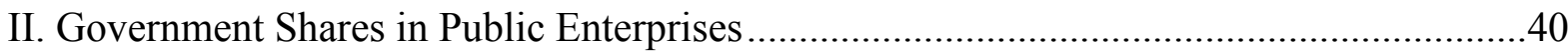

III. Summary Of The Tax System of Zambia as of October 2007 .....................................41 
Zambia: Basic Indicators

I. Socio and Demographic Indicators

Area (square km)

Arable land, in percent

Population, in million (2006)

Annual rate of population growth 2005

Crude death rate per 1000 people

Infant mortality per 1000 births (2005)
752,614 Life expectancy (2005) 38

7 Unemployment rate, in percent (2006) 14

12 Literacy rate, in percent (2005) 68

2 Primary enrollment, percent of net (2005) 89

21 GNI per capita (Atlas method, US\$, 2006) 630

II. Economic Indicators

\begin{tabular}{|c|c|c|c|c|c|}
\hline & 2002 & 2003 & 2004 & 2005 & 2006 \\
\hline & \multicolumn{5}{|c|}{ (Annual percent change) } \\
\hline Real GDP & 3.3 & 5.1 & 5.4 & 5.2 & 6.2 \\
\hline GDP deflator & 19.9 & 19.8 & 20.4 & 18.7 & 13.8 \\
\hline Consumer prices, annual average & 22.2 & 21.4 & 18.0 & 18.3 & 9.0 \\
\hline \multirow[t]{2}{*}{ Consumer prices, end of period } & 26.7 & 17.2 & 17.5 & 15.9 & 8.2 \\
\hline & \multicolumn{5}{|c|}{ (In percent of GDP) } \\
\hline \multicolumn{6}{|l|}{ Origin of GDP } \\
\hline Agriculture, forestry, and fishing & 20.0 & 20.7 & 21.4 & 20.7 & 19.9 \\
\hline Mining and quarrying & 3.5 & 2.8 & 3.1 & 3.2 & 4.1 \\
\hline Manufacturing & 10.4 & 10.9 & 10.9 & 10.6 & 10.2 \\
\hline Electricity, gas, and water & 3.0 & 2.9 & 2.7 & 2.8 & 3.0 \\
\hline Construction & 6.6 & 7.8 & 9.2 & 11.5 & 13.9 \\
\hline Services & 56.5 & 54.9 & 52.7 & 51.2 & 48.9 \\
\hline \multicolumn{6}{|l|}{ Central government } \\
\hline Total revenue and grants & 26.2 & 24.9 & 23.7 & 23.0 & 42.9 \\
\hline Total expenditure and net lending & 31.3 & 30.9 & 26.6 & 25.7 & 23.1 \\
\hline \multicolumn{6}{|l|}{ Of which: } \\
\hline Wages and salaries & 8.0 & 8.4 & 7.7 & 7.6 & 7.2 \\
\hline Interest & 4.1 & 3.9 & 3.5 & 2.7 & 1.9 \\
\hline Capital expenditure & 11.8 & 11.4 & 8.7 & 7.0 & 4.1 \\
\hline Savings & -0.6 & -1.3 & 0.7 & -0.6 & -1.5 \\
\hline Primary balance after grants & -1.5 & -2.6 & 2.7 & 0.0 & 20.5 \\
\hline Overall balance after grants $1 /$ & -5.5 & -6.5 & -0.8 & -2.6 & 18.6 \\
\hline & \multicolumn{5}{|c|}{ (Percent contribution to growth in broad money) $2 /$} \\
\hline \multicolumn{6}{|l|}{ Money and credit } \\
\hline Liabilities to nongovernment sector & 31.4 & 23.4 & 30.3 & 0.4 & 45.1 \\
\hline Money & 11.6 & 10.1 & 7.8 & 4.1 & 20.3 \\
\hline Quasi money & 19.9 & 13.3 & 22.4 & -3.7 & 24.9 \\
\hline Net domestic assets of the banking system & 43.0 & 3.4 & 19.8 & -28.6 & -27.4 \\
\hline Net credit to the general government & -3.8 & 26.6 & -4.4 & -2.2 & -0.2 \\
\hline Claims on nongovernment & -10.0 & 10.9 & 16.7 & 7.1 & 22.5 \\
\hline Other net assets & 33.4 & -34.1 & 7.5 & -33.6 & -49.7 \\
\hline Liabilities to nongovernment, in percent of GDP & 22.3 & 21.8 & 22.4 & 18.0 & 21.6 \\
\hline Treasury bill rate & 31.7 & 19.7 & 18.2 & 16.4 & 9.2 \\
\hline
\end{tabular}


Zambia: Basic Indicators (concluded)

\begin{tabular}{|c|c|c|c|c|c|}
\hline & 2002 & 2003 & 2004 & 2005 & 2006 \\
\hline & \multicolumn{5}{|c|}{ (In million of U.S. dollars, unless otherwise indicated) } \\
\hline \multicolumn{6}{|l|}{ Balance of payments } \\
\hline Current account balance & -578.5 & -640.9 & -617.6 & -661.5 & 119.7 \\
\hline Of which: Merchandise trade balance & -259.3 & -302.1 & -25.0 & 49.8 & 1183.4 \\
\hline Export of goods & 944.3 & 1090.4 & 1846.9 & 2210.4 & 3819.2 \\
\hline Import of goods & -1203.6 & -1392.5 & -1871.9 & -2160.6 & -2635.8 \\
\hline Capital and financial account & 386.7 & 420.5 & 258.5 & 704.1 & 1013.1 \\
\hline \multicolumn{6}{|l|}{ Of which: } \\
\hline Official borrowing (net) $1 /$ & -53.0 & -131.2 & -200.7 & -65.1 & -1779.0 \\
\hline Net foreign direct investment & 303.0 & 347.0 & 364.0 & 380.0 & 467.0 \\
\hline Errors and omissions & -19.5 & -7.6 & 139.0 & -171.9 & -311.2 \\
\hline Overall balance & -211.4 & -228.0 & -220.1 & -129.3 & 821.6 \\
\hline Exports of goods and nonfactor services, percent of GDP & 28.6 & 29.0 & 38.2 & 34.1 & 37.8 \\
\hline Imports of goods and nonfactor services, percent of GDP & -42.0 & -41.5 & -42.6 & -36.2 & -29.6 \\
\hline Current account, in percent of GDP & -15.3 & -14.8 & -11.4 & -9.1 & 1.1 \\
\hline Exports in US dollars, annual percent change & 22.0 & 3.7 & 15.5 & 69.4 & 19.7 \\
\hline Imports in US dollars, annual percent change & 28.2 & -3.9 & 15.7 & 34.4 & 15.4 \\
\hline Terms of trade, annual percent change & -1.9 & 1.4 & 34.6 & 6.8 & 54.8 \\
\hline Real effective exchange rate, end of period, percent change & -10.2 & 5.9 & 9.4 & 57.1 & -1.3 \\
\hline External debt, in percent of GDP & 178.7 & 156.5 & 126.7 & 86.0 & 8.8 \\
\hline Government scheduled debt service ratio $2 /$ & 54.4 & 48.7 & 34.1 & 95.9 & 59.9 \\
\hline Of which: interest 2/ & 12.2 & 10.0 & 5.8 & 4.4 & 0.4 \\
\hline \multicolumn{6}{|l|}{ IMF data as of September 30, 2007} \\
\hline Membership status: & & & & \multicolumn{2}{|c|}{ September 23, 1965} \\
\hline General resource account qouta & & & & \multicolumn{2}{|c|}{ SDR 489.1 million } \\
\hline \multicolumn{6}{|l|}{ Special drawing rights department } \\
\hline Net cumulative allocation & & & & \multicolumn{2}{|c|}{ SDR 68.3 million } \\
\hline Holdings of SDRs & & & & \multicolumn{2}{|c|}{ SDR 6.91 million } \\
\hline Outstanding purchases and loans & & & & \multicolumn{2}{|c|}{ SDR 55.02 million } \\
\hline \multicolumn{6}{|l|}{ Latest PRGF financial arrangement: } \\
\hline Date of arrangement & & & & \multicolumn{2}{|c|}{ June 16, 2004} \\
\hline Expiration date & & & & \multicolumn{2}{|c|}{ September 30,2007} \\
\hline Amount approved & & & & \multicolumn{2}{|c|}{ SDR 220.1 million } \\
\hline Amount withdrawn & & & & \multicolumn{2}{|c|}{ SDR 220.1 million } \\
\hline
\end{tabular}

Source: Zambian authorities, World Development Indicators (2007), and International Monetary Fund.

1/ Includes debt stock reduction under the IMF's Multilateral Debt Relief Initiative, SDR 402.6 million in 2006.

$2 /$ In percent of exports of goods and services. 
Table 1. Zambia: Gross Domestic Product by Sector of Origin at Constant Prices

\begin{tabular}{|c|c|c|c|c|c|}
\hline & 2002 & 2003 & 2004 & 2005 & 2006 \\
\hline & \multicolumn{5}{|c|}{ (In billions of Zambia kwacha, 1994=100) } \\
\hline Real GDP & $2,707.9$ & $2,846.5$ & $3,000.0$ & $3,156.0$ & $3,351.6$ \\
\hline Agriculture, forestry, and fishing & 411.7 & 432.5 & 450.8 & 448.3 & 458.2 \\
\hline Mining and quarrying & 212.9 & 220.2 & 250.9 & 270.8 & 290.6 \\
\hline Manufacturing & 289.4 & 311.4 & 325.9 & 335.3 & 354.6 \\
\hline Electricity, gas, and water & 77.8 & 78.1 & 76.8 & 81.0 & 89.5 \\
\hline Construction & 161.8 & 196.8 & 237.1 & 287.3 & 328.7 \\
\hline Wholesale and retail trade & 505.4 & 536.4 & 563.1 & 576.7 & 588.1 \\
\hline Transport and communications & 165.1 & 173.0 & 184.1 & 204.4 & 249.4 \\
\hline Community, social, and personal services $1 /$ & 207.3 & 210.5 & 211.7 & 235.9 & 257.0 \\
\hline Financial institutions and insurance & 212.7 & 220.0 & 227.7 & 235.1 & 244.6 \\
\hline Real estate and business services & 257.4 & 267.6 & 278.2 & 287.1 & 296.2 \\
\hline Restaurants and hotels & 62.9 & 67.2 & 71.5 & 79.9 & 92.8 \\
\hline Other & 143.5 & 132.8 & 122.2 & 114.2 & 101.9 \\
\hline Import duties & 271.6 & 264.1 & 256.9 & 252.2 & 243.5 \\
\hline Less: imputed banking service charges & 128.1 & 131.3 & 134.6 & 138.0 & 141.6 \\
\hline \multicolumn{6}{|l|}{ Memorandum items: } \\
\hline Nonagricultural value added & $2,296.2$ & $2,414.1$ & $2,549.2$ & $2,707.7$ & $2,893.4$ \\
\hline Nonmining value added & $2,495.0$ & $2,626.3$ & $2,749.2$ & $2,885.2$ & $3,061.0$ \\
\hline & \multicolumn{5}{|c|}{ (Annual percent change) } \\
\hline Real GDP & 3.3 & 5.1 & 5.4 & 5.2 & 6.2 \\
\hline Agriculture, forestry, and fishing & -1.7 & 5.0 & 4.3 & -0.6 & 2.2 \\
\hline Mining and quarrying & 16.4 & 3.4 & 13.9 & 7.9 & 7.3 \\
\hline Manufacturing & 5.7 & 7.6 & 4.7 & 2.9 & 5.8 \\
\hline Electricity, gas, and water & -5.2 & 0.4 & -1.7 & 5.5 & 10.5 \\
\hline Construction & 17.4 & 21.6 & 20.5 & 21.2 & 14.4 \\
\hline Wholesale and retail trade & 5.0 & 6.1 & 5.0 & 2.4 & 2.0 \\
\hline Transport and communications & 1.8 & 4.8 & 6.4 & 11.1 & 22.0 \\
\hline Community, social, and personal services $1 /$ & 1.6 & 1.6 & 0.6 & 11.4 & 8.9 \\
\hline Financial institutions and insurance & 3.5 & 3.5 & 3.5 & 3.3 & 4.0 \\
\hline Real estate and business services & 4.4 & 4.0 & 4.0 & 3.2 & 3.2 \\
\hline Restaurants and hotels & 4.9 & 6.9 & 6.4 & 11.7 & 16.1 \\
\hline Other & -13.8 & -7.5 & -7.9 & -6.6 & -10.8 \\
\hline Import duties & -6.8 & -2.8 & -2.7 & -1.8 & -3.4 \\
\hline Less: imputed banking service charges & 2.5 & 2.5 & 2.5 & 2.5 & 2.6 \\
\hline \multicolumn{6}{|l|}{ Memorandum items: } \\
\hline Nonagricultural value added & 4.3 & 5.1 & 5.6 & 6.2 & 6.9 \\
\hline Nonmining value added & 2.3 & 5.3 & 4.7 & 4.9 & 6.1 \\
\hline
\end{tabular}

Source: Central Statistical Office.

1/ Includes public administration, defense, sanitary services, education, health, recreation, and personal services. 
Table 2. Zambia: Gross Domestic Product by Sector of Origin at Current Prices

\begin{tabular}{|c|c|c|c|c|c|}
\hline & 2002 & 2003 & 2004 & 2005 & 2006 \\
\hline & \multicolumn{5}{|c|}{ (In billions of Zambia kwacha) } \\
\hline Nominal GDP at market prices & $16,260.4$ & $20,479.2$ & $25,997.4$ & $32,456.2$ & $39,223.1$ \\
\hline Agriculture, forestry, and fishing & $3,247.4$ & $4,244.6$ & $5,568.2$ & 6723.6 & 7,800 \\
\hline Mining and quarrying & 575.1 & 564.8 & 809.6 & 1030.9 & 1,613 \\
\hline Manufacturing & $1,693.6$ & $2,241.0$ & $2,827.7$ & 3430.2 & 4,016 \\
\hline Electricity, gas, and water & 488.3 & 595.1 & 694.7 & 922.7 & 1,166 \\
\hline Construction & $1,067.7$ & $1,590.0$ & $2,402.1$ & 3728 & 5,463 \\
\hline Wholesale and retail trade & $3,004.1$ & $3,873.8$ & $4,843.7$ & 5868.9 & 6,525 \\
\hline Transport and communications & $1,055.9$ & $1,058.2$ & $1,252.3$ & 1395.6 & 1,629 \\
\hline Community, social, and personal services $1 /$ & $1,414.4$ & $1,757.0$ & $2,046.5$ & 2710 & 3,366 \\
\hline Financial institutions and insurance & $1,493.1$ & $1,847.7$ & $2,282.7$ & 2771.5 & 3,247 \\
\hline Real estate and business services & $1,041.2$ & $1,341.2$ & $1,691.8$ & 1979.4 & 2,296 \\
\hline Restaurants and hotels & 406.8 & 527.7 & 670.9 & 894 & 1,120 \\
\hline Other & 772.8 & 838.1 & 907.3 & $1,001.4$ & 983.3 \\
\hline Import duties & $1,630.8$ & $1,899.9$ & $2,219.1$ & 2594.2 & 2,849 \\
\hline Less: imputed banking service charges & 858.1 & $1,061.8$ & $1,311.8$ & 1592.8 & 1,866 \\
\hline \multirow[t]{2}{*}{ GDP deflator } & 600.5 & 719.4 & 866.6 & $1,028.4$ & $1,170.3$ \\
\hline & \multicolumn{5}{|c|}{ (In percent of GDP unless otherwise indicated) } \\
\hline Nominal GDP, annual percent change & 23.8 & 25.9 & 26.9 & 24.8 & 20.8 \\
\hline Agriculture, forestry, and fishing & 20.0 & 20.7 & 21.4 & 20.7 & 19.9 \\
\hline Mining and quarrying & 3.5 & 2.8 & 3.1 & 3.2 & 4.1 \\
\hline Manufacturing & 10.4 & 10.9 & 10.9 & 10.6 & 10.2 \\
\hline Electricity, gas, and water & 3.0 & 2.9 & 2.7 & 2.8 & 3.0 \\
\hline Construction & 6.6 & 7.8 & 9.2 & 11.5 & 13.9 \\
\hline Wholesale and retail trade & 18.5 & 18.9 & 18.6 & 18.1 & 16.6 \\
\hline Transport and communications & 6.5 & 5.2 & 4.8 & 4.3 & 4.2 \\
\hline Community, social, and personal services $1 /$ & 8.7 & 8.6 & 7.9 & 8.3 & 8.6 \\
\hline Financial institutions and insurance & 9.2 & 9.0 & 8.8 & 8.5 & 8.3 \\
\hline Real estate and business services & 6.4 & 6.5 & 6.5 & 6.1 & 5.9 \\
\hline Restaurants and hotels & 2.5 & 2.6 & 2.6 & 2.8 & 2.9 \\
\hline Other & 4.8 & 4.1 & 3.5 & 3.1 & 2.5 \\
\hline Import duties & 10.0 & 9.3 & 8.5 & 8.0 & 7.3 \\
\hline Less: imputed banking service charges & 5.3 & 5.2 & 5.0 & 4.9 & 4.8 \\
\hline
\end{tabular}

Source: Central Statistical Office.

1/ Includes public administration, defense, sanitary services, education, health, recreation, and personal services. 
Table 3. Zambia: Index of Industrial Production

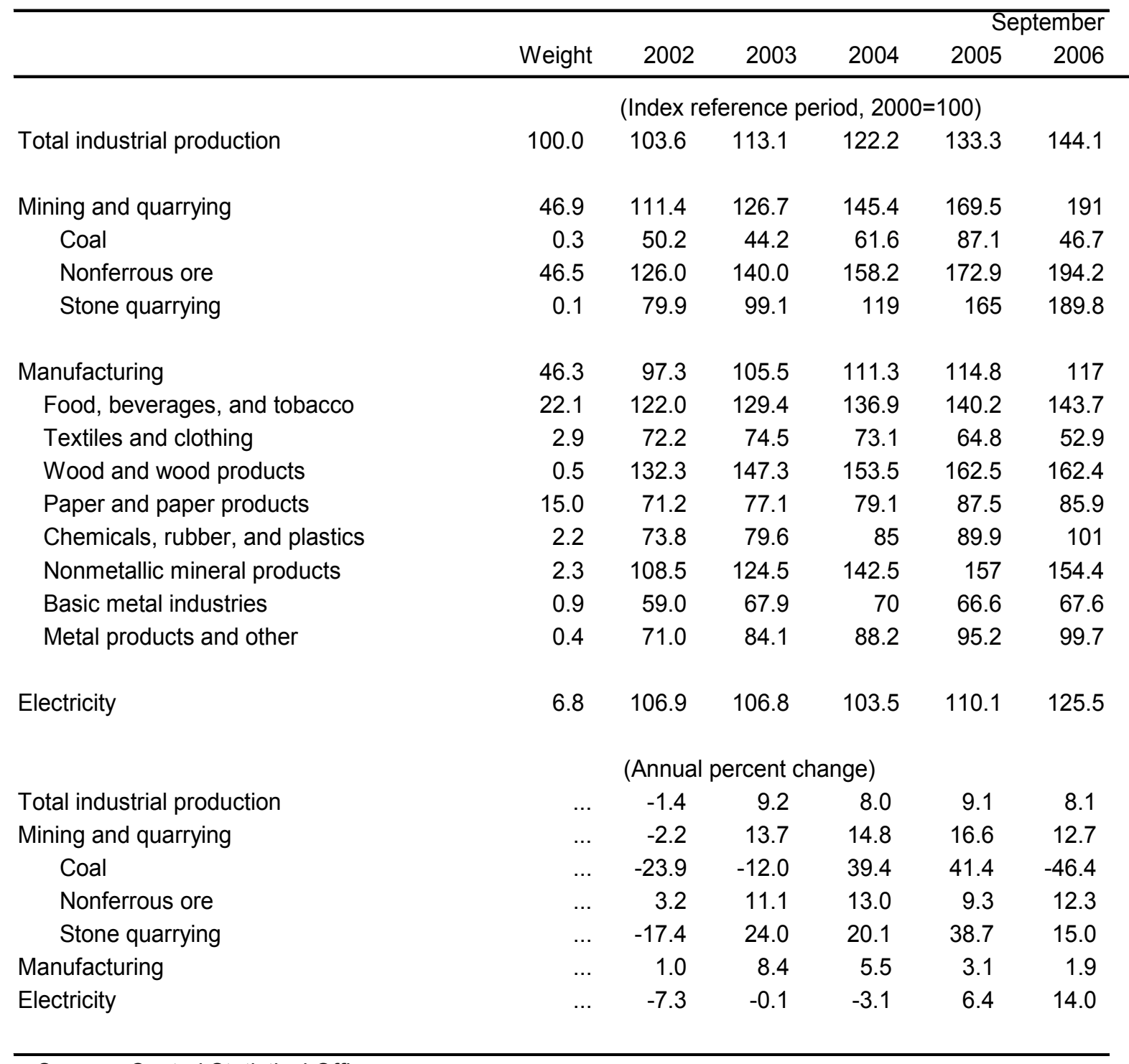

Source: Central Statistical Office. 
Table 4. Zambia: Volume of Mineral Production

(In thousands of metric tons)

\begin{tabular}{lrrrrrr}
\hline & 2002 & 2003 & 2004 & 2005 & 2006 \\
\hline Coal & 71.7 & 71.8 & 103.1 & 148.8 & $\ldots$ \\
Cobalt & 3.9 & 3.2 & 6.1 & 5.5 & 4.7 \\
Copper & 337.7 & 353.4 & 419.6 & 459.3 & 498.3 \\
\hline
\end{tabular}

Source: Ministry of Mines. 
Table 5. Zambia: Marketed Production of Selected Agricultural Crops 1/

\begin{tabular}{|c|c|c|c|c|c|}
\hline & $2001 / 02$ & $2002 / 03$ & $2003 / 04$ & $2004 / 05$ & $\begin{array}{r}\text { Prel. } \\
2005 / 06\end{array}$ \\
\hline & \multicolumn{4}{|c|}{ (In metric tons) } & \\
\hline Maize & 112,382 & 591,300 & 481,183 & 349,734 & $1,110,919$ \\
\hline Tobacco (Virginia) & 915 & $\ldots$ & 12,355 & 7,246 & 2,097 \\
\hline Tobacco (burley) & 4,920 & 27,278 & 9,308 & 137 & 7,412 \\
\hline Mixed beans & 7,541 & 10,921 & 8,455 & 12,258 & 27,039 \\
\hline Groundnuts & 19,878 & 29,946 & 21,249 & 26,678 & 83,777 \\
\hline Sunflower seeds & 4,722 & 1,227 & 9,367 & 3,391 & 14,825 \\
\hline Seed cotton & 64,659 & 389,591 & 141,228 & 2,136 & 117,811 \\
\hline Wheat & 4,722 & 135,287 & 77,740 & 133,302 & 142,347 \\
\hline Paddy rice & 6,189 & 5,716 & 3,524 & 6,626 & 13,964 \\
\hline Soya beans & 1,756 & 41,410 & 48,910 & 81,673 & 18,785 \\
\hline \multirow[t]{2}{*}{ Sorghum } & 1,385 & 2,625 & 2,519 & 2,304 & 18,465 \\
\hline & \multicolumn{5}{|c|}{ (Index reference period: $1990 / 91=100)$} \\
\hline Maize & 19 & 98 & 80 & 58 & 184 \\
\hline Tobacco (Virginia) & 106 & $\ldots$ & 1,430 & 839 & 243 \\
\hline Tobacco (burley) & 607 & 3,368 & 1,149 & 17 & 915 \\
\hline Mixed beans & 125 & 181 & 140 & 204 & 449 \\
\hline Groundnuts & 225 & 338 & 240 & 301 & 947 \\
\hline Sunflower seeds & 31 & 8 & 62 & 22 & 97 \\
\hline Seed cotton & 133 & 800 & 290 & 4 & 242 \\
\hline Wheat & 9 & 264 & 152 & 260 & 278 \\
\hline Paddy rice & 70 & 64 & 40 & 74 & 157 \\
\hline Soya beans & 7 & 170 & 201 & 335 & 77 \\
\hline Sorghum & 138 & 261 & 250 & 229 & 1,834 \\
\hline
\end{tabular}

Source: Central Statistical Office.

1/ Crop years run from May 1 to April 30 . 
Table 6. Zambia: Area Under Cultivation for Selected Crops 1/

\begin{tabular}{|c|c|c|c|c|c|}
\hline & $2001 / 02$ & $2002 / 032 /$ & $2003 / 04$ & $2004 / 05$ & $\begin{array}{r}\text { Prel. } \\
2005 / 06\end{array}$ \\
\hline & \multicolumn{4}{|c|}{ (In hectares) } & \\
\hline Total area & $1,065,195$ & $1,157,955$ & $1,116,612$ & $1,497,056$ & $1,324,539$ \\
\hline Maize & 646,450 & 699,276 & 631,079 & 834,981 & 732,917 \\
\hline Groundnuts & 139,015 & 150,460 & 116,978 & 161,962 & 143,803 \\
\hline Sunflower seeds & 22,139 & 22,521 & 30,689 & 31,191 & 39,038 \\
\hline Cotton & 87,026 & 86,431 & 121,593 & 176,217 & 151,618 \\
\hline Soya beans & 6,820 & 17,402 & 33,186 & 65,170 & 26,511 \\
\hline Tobacco & 3,855 & 11,204 & 5,464 & 15,630 & 8,834 \\
\hline Paddy rice & 13,050 & 10,305 & 12,379 & 18,243 & 14,358 \\
\hline Sorghum & 33,955 & 37,054 & 47,350 & 57,432 & 42,368 \\
\hline Millet & 61,347 & 56,751 & 59,081 & 63,411 & 67,788 \\
\hline Mixed beans & 40,043 & 44,002 & 45,270 & 50,496 & 53,697 \\
\hline \multirow[t]{2}{*}{ Other include wheat } & 11,495 & 22,549 & 13,543 & 22,323 & 43,606 \\
\hline & \multicolumn{4}{|c|}{ (Annual percent change) } & \\
\hline Total area & 4.9 & 8.7 & -3.6 & 34.1 & -11.5 \\
\hline Maize & 10.7 & 8.2 & -9.8 & 32.3 & -12.2 \\
\hline Groundnuts & 1.4 & 8.2 & -22.3 & 38.5 & -11.2 \\
\hline Sunflower seeds & -40.8 & 1.7 & 36.3 & 1.6 & 25.2 \\
\hline Cotton & 52.9 & -0.7 & 40.7 & 44.9 & -14.0 \\
\hline Soya beans & -59.3 & 155.2 & 90.7 & 96.4 & -59.3 \\
\hline Tobacco & -9.2 & 190.6 & -51.2 & 186.1 & -43.5 \\
\hline Paddy rice & -8.9 & -21.0 & 20.1 & 47.4 & -21.3 \\
\hline Sorghum & -21.7 & 9.1 & 27.8 & 21.3 & -26.2 \\
\hline Millet & -12.5 & -7.5 & 4.1 & 7.3 & 6.9 \\
\hline Mixed beans & -21.5 & 9.9 & 2.9 & 11.5 & 6.3 \\
\hline Other include wheat & $\ldots$ & 96.2 & -39.9 & 64.8 & 95.3 \\
\hline
\end{tabular}

Sources: Ministry of Agriculture and Co-operatives; and Central Statistical Office.

$1 /$ Crop years run from May 1 to April 30. Data are based on Post Harvest Survey results.

2/ 2003 data based on the Final Crop Forecasting Survey for 2003. 
Table 7. Zambia: Paid Employment by Economic Sector

\begin{tabular}{|c|c|c|c|c|c|}
\hline & 2002 & 2003 & 2004 & $20051 /$ & 2006 \\
\hline & \multicolumn{5}{|c|}{ (In number of employees $2 /$ ) } \\
\hline All sectors & 429,406 & 416,804 & 416,228 & $\ldots$ & 479,327 \\
\hline Agriculture, forestry, and fishing & 43,819 & 64,096 & 65,136 & $\ldots$ & 56,139 \\
\hline Mining and quarrying & 37,245 & 48,597 & 46,078 & $\ldots$ & 26,253 \\
\hline Manufacturing & 67,752 & 39,385 & 45,340 & $\ldots$ & 55,709 \\
\hline Electricity and water & 7,316 & 10,832 & 12,346 & $\ldots$ & 12,399 \\
\hline Construction & 2,406 & 3,467 & 5,787 & $\ldots$ & 14,343 \\
\hline Transport and communications & 21,566 & 26,725 & 26,510 & $\ldots$ & 19,378 \\
\hline Distribution and trade (wholesale and retail) & 50,812 & 53,450 & 44,460 & $\ldots$ & 65,012 \\
\hline Finance and insurance & 52,727 & 28,555 & 31,880 & $\ldots$ & 54,032 \\
\hline \multirow[t]{2}{*}{ Public administration } & 145,763 & 141,697 & 138,691 & $\ldots$ & 176,062 \\
\hline & \multicolumn{5}{|c|}{ (Simple average earnings by industry in thousand kwacha $2 /$ ) } \\
\hline All sectors & 678 & 680 & 809 & $\cdots$ & 1,472 \\
\hline Agriculture, forestry, and fishing & 223 & 222 & 267 & $\ldots$ & 382 \\
\hline Mining and quarrying & 548 & 570 & 839 & $\cdots$ & 2,936 \\
\hline Manufacturing & 791 & 722 & 1,314 & $\ldots$ & 1,440 \\
\hline Electricity and water & 1,044 & 1,327 & 1,620 & $\ldots$ & 5,645 \\
\hline Construction & 342 & 93 & 611 & $\ldots$ & 862 \\
\hline Transport and communications & 833 & 1,608 & 1,137 & $\ldots$ & 693 \\
\hline Distribution and trade (wholesale and retail) & 401 & 381 & 682 & $\ldots$ & 1,580 \\
\hline Finance and insurance & 431 & 992 & 1,289 & $\ldots$ & 3,160 \\
\hline \multirow[t]{2}{*}{ Public administration } & 376 & 376 & 694 & $\cdots$ & 963 \\
\hline & \multicolumn{5}{|c|}{ (Annual percent change) } \\
\hline Number of employees & -9.7 & -2.9 & -0.1 & $\ldots$ & $\cdots$ \\
\hline Earnings (simple average) & $\ldots$ & 0.3 & 19.0 & $\ldots$ & $\ldots$ \\
\hline
\end{tabular}

Source: Central Statistical Office (Labor force survey).

1/ There was no labor force survey in 2005.

2/ This period refers to January. 
Table 8. Zambia: Annual Average Composite Index of Consumer Prices

\begin{tabular}{|c|c|c|c|c|c|c|}
\hline & Weights & 2002 & 2003 & 2004 & 2005 & 2006 \\
\hline & \multicolumn{6}{|c|}{$1994=100$} \\
\hline Composite index $1 /$ & 1,000 & 708.8 & 860.5 & 1015.1 & 1201.1 & 1309.4 \\
\hline Food and beverages index & 571 & 661.6 & 808.0 & 940.2 & 1114.8 & 1169.7 \\
\hline Nonfood composite index & 429 & 771.3 & 930.1 & 1114.6 & 1316.0 & 1495.0 \\
\hline Clothing and footwear & 68 & 753.4 & 916.3 & 1160.2 & 1326.7 & 1694.4 \\
\hline Rent, fuel, and lighting & 85 & 824.6 & 999.8 & 1170.5 & 1474.7 & 1685.9 \\
\hline Furniture and household goods & 82 & 744.0 & 940.4 & 1191.3 & 1433.0 & 1661.2 \\
\hline Medical care & 8 & 770.9 & 889.1 & 1041.5 & 1183.3 & 1391.3 \\
\hline Transport and communication & 96 & 747.4 & 874.7 & 979.8 & 1123.4 & 1102.1 \\
\hline Recreation and education & 49 & 1000.3 & 1172.0 & 1407.2 & 1593.3 & 1818.9 \\
\hline \multirow[t]{2}{*}{ All other goods and services } & 41 & 527.3 & 636.1 & 748.8 & 880.4 & 990.3 \\
\hline & \multicolumn{4}{|c|}{ (Annual percent change) } & & \\
\hline Composite index $1 /$ & $\ldots$ & 22.2 & 21.4 & 18.0 & 18.3 & 9.0 \\
\hline Food, beverages and tobacco & $\ldots$ & 27.1 & 22.1 & 16.4 & 18.6 & 4.9 \\
\hline Nonfood composite index & $\ldots$ & 17.1 & 20.6 & 19.8 & 18.1 & 13.6 \\
\hline Clothing and footwear & $\ldots$ & 19.5 & 21.6 & 26.6 & 14.4 & 27.7 \\
\hline Rent, fuel, and lighting & $\ldots$ & 15.7 & 21.2 & 17.1 & 26.0 & 14.3 \\
\hline Furniture and household goods & $\ldots$ & 22.6 & 26.4 & 26.7 & 20.3 & 15.9 \\
\hline Medical care & $\ldots$ & 18.4 & 15.3 & 17.1 & 13.6 & 17.6 \\
\hline Transport and communication & $\ldots$ & 8.3 & 17.0 & 12.0 & 14.7 & -1.9 \\
\hline Recreation and education & $\ldots$ & 24.9 & 17.2 & 20.1 & 13.2 & 14.2 \\
\hline All other goods and services & $\ldots$ & 16.5 & 20.6 & 17.7 & 17.6 & 12.5 \\
\hline
\end{tabular}

Source: Central Statistical Office.

1/ Composite index consists of food and nonfood indices. 
Table 9. Zambia: Monthly Composite Index of Consumer Prices (Index reference period: 1994=100)

\begin{tabular}{|c|c|c|c|c|c|c|c|c|c|c|}
\hline & & & & & & Furniture \& & & Transport & Recreation & All other \\
\hline Weights & $\begin{array}{c}\text { Composite } \\
\text { Index 1/ } \\
1000\end{array}$ & $\begin{array}{c}\text { Food \& } \\
\text { Beverages } \\
571\end{array}$ & $\begin{array}{c}\text { Nonfood } \\
\text { Composite } \\
429\end{array}$ & $\begin{array}{c}\text { Clothing \& } \\
\text { Footwear } \\
68\end{array}$ & $\begin{array}{l}\text { Rent, Fuel } \\
\text { \& Lighting } \\
\quad 85\end{array}$ & $\begin{array}{c}\text { Household } \\
\text { Goods } \\
82\end{array}$ & $\begin{array}{c}\text { Medical } \\
\text { Care } \\
8\end{array}$ & $\begin{array}{c}\text { \& Commu- } \\
\text { nication } \\
96\end{array}$ & $\begin{array}{c}\text { and } \\
\text { Education } \\
49\end{array}$ & $\begin{array}{c}\text { Goods \& } \\
\text { Services } \\
41\end{array}$ \\
\hline 2005 & $1,201.1$ & $1,114.8$ & $1,316.0$ & $1,326.7$ & $1,474.7$ & $1,433.0$ & $1,183.3$ & $1,123.4$ & $1,593.3$ & 880.4 \\
\hline January & $1,134.3$ & $1,059.3$ & $1,233.8$ & $1,244.3$ & $1,318.4$ & $1,328.3$ & $1,132.6$ & $1,099.9$ & $1,528.3$ & 833.0 \\
\hline February & $1,151.6$ & $1,072.5$ & $1,259.5$ & $1,281.5$ & $1,371.3$ & $1,348.7$ & 1,143.6 & $1,113.5$ & $1,537.7$ & 844.9 \\
\hline March & $1,156.1$ & $1,072.0$ & $1,267.8$ & $1,275.6$ & $1,379.8$ & $1,367.1$ & 1,151.6 & $1,117.9$ & $1,555.4$ & 853.6 \\
\hline April & $1,171.5$ & $1,086.6$ & $1,284.1$ & $1,280.2$ & $1,420.3$ & $1,392.3$ & $1,154.2$ & $1,122.5$ & $1,563.7$ & 861.6 \\
\hline May & $1,184.7$ & $1,098.6$ & $1,299.2$ & $1,296.9$ & $1,432.5$ & $1,398.4$ & $1,159.5$ & $1,143.4$ & $1,592.1$ & 870.4 \\
\hline June & $1,194.8$ & $1,102.4$ & $1,317.4$ & $1,314.6$ & $1,487.3$ & $1,419.3$ & $1,174.5$ & $1,140.8$ & $1,594.4$ & 877.2 \\
\hline July & $1,196.6$ & 100.9 & $1,323.9$ & $1,319.7$ & 8 & $1,437.4$ & $1,186.9$ & $1,128.2$ & $1,597.5$ & 886.4 \\
\hline August & $1,214.4$ & $1,126.1$ & $1,331.6$ & $1,338.2$ & $1,515.0$ & $1,443.3$ & $1,209.1$ & $1,128.6$ & $1,601.1$ & 894.6 \\
\hline September & $1,231.2$ & $1,136.1$ & $1,357.6$ & $1,354.7$ & $1,528.3$ & $1,496.4$ & $1,213.4$ & $1,162.9$ & $1,622.9$ & 897.5 \\
\hline October & $1,244.1$ & $1,148.9$ & $1,370.5$ & $1,381.6$ & $1,562.4$ & $1,506.5$ & $1,217.7$ & $1,153.5$ & $1,633.9$ & 905.4 \\
\hline November & $1,260.6$ & $1,172.9$ & $1,377.1$ & $1,406.1$ & $1,583.0$ & $1,520.3$ & $1,227.5$ & $1,124.5$ & $1,646.0$ & 914.6 \\
\hline $\begin{array}{r}\text { December } \\
2006\end{array}$ & $1,273.2$ & $1,200.9$ & $1,369.3$ & $1,427.5$ & $1,593.8$ & $1,538.1$ & $1,228.6$ & $1,044.5$ & $1,646.9$ & 925.5 \\
\hline January & $1,272.3$ & $1,194.5$ & $1,375.7$ & $1,438.5$ & $1,587.4$ & $1,570.4$ & $1,248.6$ & $1,005.3$ & $1,714.2$ & 930.7 \\
\hline February & $1,270.0$ & $1,182.1$ & $1,386.5$ & $1,537.6$ & $1,583.7$ & $1,532.9$ & $1,264.0$ & $1,002.9$ & $1,732.8$ & 942.9 \\
\hline March & $1,279.6$ & $1,189.3$ & $1,399.3$ & $1,568.5$ & $1,616.5$ & $1,536.9$ & $1,284.4$ & 999.3 & $1,738.3$ & 947.1 \\
\hline April & $1,281.6$ & $1,176.7$ & $1,420.7$ & $1,612.0$ & $1,629.5$ & $1,571.4$ & $1,327.2$ & $1,000.5$ & $1,747.3$ & 981.0 \\
\hline May & $1,286.8$ & $1,159.7$ & $1,455.3$ & $1,676.0$ & $1,634.9$ & $1,603.4$ & $1,355.7$ & $1,034.9$ & $1,817.8$ & 991.7 \\
\hline June & $1,296.8$ & $1,160.8$ & $1,477.2$ & $1,681.5$ & $1,645.0$ & $1,653.7$ & $1,380.4$ & $1,067.0$ & $1,827.6$ & 997.5 \\
\hline July & $1,300.2$ & $1,147.9$ & $1,504.2$ & $1,704.6$ & $1,688.3$ & $1,671.4$ & $1,393.6$ & $1,111.2$ & $1,838.4$ & 998.6 \\
\hline August & $1,311.1$ & $1,131.1$ & $1,549.9$ & $1,732.0$ & $1,744.9$ & $1,723.7$ & $1,440.8$ & $1,197.7$ & $1,841.8$ & $1,007.8$ \\
\hline September & $1,332.6$ & $1,152.7$ & $1,571.3$ & $1,763.6$ & $1,755.3$ & $1,739.6$ & $1,468.9$ & $1,218.9$ & $1,876.3$ & $1,014.7$ \\
\hline October & $1,341.8$ & $1,160.7$ & $1,582.0$ & $1,851.4$ & $1,754.1$ & $1,752.4$ & $1,481.5$ & $1,184.2$ & $1,891.0$ & $1,019.5$ \\
\hline November & $1,362.2$ & $1,182.4$ & $1,600.7$ & $1,863.4$ & $1,789.4$ & $1,773.6$ & $1,512.8$ & $1,201.1$ & $1,899.3$ & $1,023.8$ \\
\hline December & $1,378.1$ & $1,198.1$ & $1,616.9$ & $1,903.8$ & $1,801.2$ & $1,804.6$ & $1,537.5$ & $1,202.7$ & $1,902.0$ & $1,027.8$ \\
\hline
\end{tabular}


Table 9. Zambia: Monthly Composite Index of Consumer Prices, 1994=100 (concluded) (12-month percent change)

\begin{tabular}{|c|c|c|c|c|c|c|c|c|c|c|}
\hline Weights & $\begin{array}{c}\text { Composite } \\
\text { Index 1/ } \\
1000\end{array}$ & $\begin{array}{c}\text { Food \& } \\
\text { Beverages } \\
571\end{array}$ & $\begin{array}{c}\text { Nonfood } \\
\text { Composite } \\
429\end{array}$ & $\begin{array}{c}\text { Clothing \& } \\
\text { Footwear } \\
68\end{array}$ & $\begin{array}{c}\text { Rent, Fuel } \\
\text { \& Lighting } \\
85\end{array}$ & $\begin{array}{c}\text { Furniture \& } \\
\text { Household } \\
\text { Goods } \\
82\end{array}$ & $\begin{array}{c}\text { Medical } \\
\text { Care } \\
8\end{array}$ & $\begin{array}{l}\text { Transport } \\
\text { \& Commu- } \\
\text { nication } \\
96\end{array}$ & $\begin{array}{c}\text { Recreation } \\
\text { and } \\
\text { Education } \\
49\end{array}$ & $\begin{array}{c}\text { All other } \\
\text { Goods \& } \\
\text { Services } \\
41\end{array}$ \\
\hline \multicolumn{11}{|l|}{2005} \\
\hline January & 18.2 & 17.9 & 18.6 & 17.3 & 18.6 & 21.7 & 12.2 & 20.6 & 13.9 & 19.6 \\
\hline February & 18.7 & 18.3 & 19.4 & 16.1 & 22.7 & 22.1 & 13.1 & 21.6 & 12.7 & 19.4 \\
\hline March & 17.4 & 16.0 & 19.0 & 14.1 & 22.1 & 22.0 & 13.2 & 22.5 & 12.7 & 18.5 \\
\hline April & 18.6 & 18.0 & 19.3 & 12.9 & 25.0 & 22.4 & 13.0 & 21.6 & 11.9 & 19.2 \\
\hline May & 19.1 & 19.1 & 19.1 & 13.0 & 25.0 & 19.4 & 12.9 & 23.1 & 13.7 & 18.0 \\
\hline June & 19.2 & 19.3 & 19.2 & 13.6 & 27.3 & 20.0 & 13.4 & 19.7 & 13.6 & 17.9 \\
\hline July & 18.7 & 18.7 & 18.7 & 13.2 & 27.9 & 21.0 & 14.2 & 15.9 & 13.4 & 17.9 \\
\hline August & 19.3 & 20.3 & 18.1 & 14.2 & 28.8 & 18.8 & 15.9 & 13.4 & 13.5 & 17.7 \\
\hline September & 19.5 & 20.7 & 18.2 & 13.9 & 28.4 & 20.8 & 15.1 & 13.1 & 12.9 & 16.3 \\
\hline October & 18.3 & 18.8 & 17.7 & 15.1 & 29.9 & 20.1 & 15.3 & 8.7 & 13.5 & 17.0 \\
\hline November & 17.2 & 18.3 & 16.1 & 14.4 & 28.9 & 18.0 & 14.5 & 4.9 & 13.5 & 15.2 \\
\hline December & 15.9 & 17.5 & 13.9 & 14.9 & 26.5 & 18.0 & 10.5 & -3.5 & 13.4 & 14.9 \\
\hline \multicolumn{11}{|l|}{2006} \\
\hline January & 12.2 & 12.8 & 11.5 & 15.6 & 20.4 & 18.2 & 10.2 & -8.6 & 12.2 & 11.7 \\
\hline February & 10.3 & 10.2 & 10.1 & 20.0 & 15.5 & 13.7 & 10.5 & -9.9 & 12.7 & 11.6 \\
\hline March & 10.7 & 10.9 & 10.4 & 23.0 & 17.2 & 12.4 & 11.5 & -10.6 & 11.8 & 11.0 \\
\hline April & 9.4 & 8.3 & 10.6 & 25.9 & 14.7 & 12.9 & 15.0 & -10.9 & 11.7 & 13.9 \\
\hline May & 8.6 & 5.6 & 12.0 & 29.2 & 14.1 & 14.7 & 16.9 & -9.5 & 14.2 & 13.9 \\
\hline June & 8.5 & 5.3 & 12.1 & 27.9 & 10.6 & 16.5 & 17.5 & -6.5 & 14.6 & 13.7 \\
\hline July & 8.7 & 4.3 & 13.6 & 29.2 & 12.2 & 16.3 & 17.4 & -1.5 & 15.1 & 12.7 \\
\hline August & 8.0 & 0.4 & 16.4 & 29.4 & 15.2 & 19.4 & 19.2 & 6.1 & 15.0 & 12.7 \\
\hline September & 8.2 & 1.5 & 15.7 & 30.2 & 14.9 & 16.3 & 21.1 & 4.8 & 15.6 & 13.1 \\
\hline October & 7.9 & 1.0 & 15.4 & 34.0 & 12.3 & 16.3 & 21.7 & 2.7 & 15.7 & 12.6 \\
\hline November & 8.1 & 0.8 & 16.2 & 32.5 & 13.0 & 16.7 & 23.2 & 6.8 & 15.4 & 11.9 \\
\hline December & 8.2 & -0.2 & 18.1 & 33.4 & 13.0 & 17.3 & 25.1 & 15.1 & 15.5 & 11.1 \\
\hline
\end{tabular}

Source: Central Statistical Office.

1/ Composite index consists of food and nonfood indices in metropolitan high and low incomes, and nonmetropolitan indices. 
Table 10. Zambia: Fuel Prices

\begin{tabular}{|c|c|c|c|c|c|}
\hline & 2002 & 2003 & 2004 & 2005 & 2006 \\
\hline & \multicolumn{5}{|c|}{ (Kwacha per liter) } \\
\hline \multicolumn{6}{|l|}{ Benchmark pump prices, end of period } \\
\hline Premium & 3,526 & 5,120 & 4,905 & 5,121 & 5,397 \\
\hline Diesel & 2,910 & 4,598 & 4,415 & 4,774 & 4,965 \\
\hline Kerosene & 2,350 & 3,639 & 3,431 & 3,505 & 3,919 \\
\hline \multicolumn{6}{|l|}{ Indeni wholesale prices, end of period } \\
\hline Premium & $\ldots$ & $\ldots$ & 2,080 & 2,073 & 2,253 \\
\hline Diesel & $\ldots$ & $\ldots$ & 2,240 & 2,357 & 2,538 \\
\hline Kerosene & $\ldots$ & $\ldots$ & 2,311 & 2,227 & 2,569 \\
\hline \multirow[t]{2}{*}{ Jet $A-1$} & $\ldots$ & $\ldots$ & 2,311 & 2,071 & 2,515 \\
\hline & \multicolumn{5}{|c|}{ (Annual percent change) } \\
\hline \multicolumn{6}{|l|}{ Benchmark pump prices, end of period } \\
\hline Premium & $\ldots$ & 45.2 & -4.2 & 4.4 & 5.4 \\
\hline Diesel & $\ldots$ & 58.0 & -4.0 & 8.1 & 4.0 \\
\hline Kerosene & $\ldots$ & 54.9 & -5.7 & 2.2 & 11.8 \\
\hline \multicolumn{6}{|l|}{ Indeni wholesale prices, end of period } \\
\hline Premium & $\ldots$ & $\ldots$ & $\ldots$ & -0.3 & 8.7 \\
\hline Diesel & $\ldots$ & $\ldots$ & $\ldots$ & 5.2 & 7.7 \\
\hline Kerosene & $\ldots$ & $\ldots$ & $\ldots$ & -3.6 & 15.4 \\
\hline Jet $A-1$ & $\ldots$ & $\ldots$ & $\ldots$ & -10.4 & 21.4 \\
\hline \multicolumn{6}{|l|}{ Memorandum items: } \\
\hline Heavy fuel oil, kwacha per 1000 metric tonne & $\ldots$ & $\ldots$ & $\ldots$ & 1,620 & 1,540 \\
\hline Heavy fuel oil, US\$ per metric tonne & $\ldots$ & $\ldots$ & $\ldots$ & 370 & 385 \\
\hline \multirow{2}{*}{$\begin{array}{l}\text { Petroleum spot price (APSP), US\$ per barrel 1/ } \\
\text { (Annual percent change) }\end{array}$} & 25.0 & 28.9 & 37.8 & 53.4 & 64.3 \\
\hline & 2.5 & 15.8 & 30.7 & 41.3 & 20.5 \\
\hline
\end{tabular}

Source: Energy Regulation Board.

1/ The Commodities Unit's APSP is a simple average of UK Brent, Dubai, and West Texas Intermediate spot prices reflecting world exports of light, medium, and heavy crude oil. 
Table 11. Zambia: Electricity Generation and Consumption 1/

\begin{tabular}{|c|c|c|c|c|c|}
\hline & 2002 & 2003 & 2004 & 2005 & 2006 \\
\hline & \multicolumn{5}{|c|}{ (In thousand megawatt-hour) } \\
\hline Total generated electricity $1 /$ & 9,168 & 8,289 & 8,448 & 8,255 & 8,906 \\
\hline Major hydros & 9,117 & 8,230 & 8,383 & 8,192 & 8,855 \\
\hline Mini hydros & 34 & 42 & 50 & 48 & 39 \\
\hline Diesel & 17 & 17 & 15 & 15 & 12 \\
\hline Domestic consumption & 6,096 & 6,617 & 7,039 & 7,452 & 7,198 \\
\hline Bulk purchases 2/ & 3,519 & 3,707 & 3,870 & 3,932 & 3,809 \\
\hline Retail domestic electricity consumed 2/ & 2,578 & 2,909 & 3,168 & 3,520 & 3,389 \\
\hline Agriculture & 73 & 83 & 119 & 107 & 158 \\
\hline Construction & 3 & 5 & 3 & 2 & 21 \\
\hline Manufacturing & 21 & 23 & 25 & 30 & 611 \\
\hline Mining and quarrying & 22 & 26 & 24 & 23 & 186 \\
\hline Other & 2,460 & 2,772 & 2,998 & 3,358 & 2,414 \\
\hline Implied exported electricity & 2,778 & 1,383 & 1,148 & 539 & 1,386 \\
\hline \multirow[t]{2}{*}{ Transmission losses } & 293 & 290 & 262 & 264 & 322 \\
\hline & \multicolumn{5}{|c|}{ (In kwacha unless otherwise indicated) } \\
\hline \multicolumn{6}{|l|}{ Energy charges, end of period $3 /$} \\
\hline Residential, above $700 \mathrm{kWh}$ & 140 & 147 & 147 & 163 & 163 \\
\hline Commercial & 140 & 147 & 147 & 163 & 163 \\
\hline Maximum demand, upto $300 \mathrm{kVa}$ & 5,939 & 6,225 & 6,225 & 6,943 & 6,943 \\
\hline Energy charge/kWh & 85 & 90 & 90 & 100 & 100 \\
\hline Fixed monthly charge & 58,165 & 61,263 & 61,263 & 68,001 & 68,002 \\
\hline Maximum demand , 301-2000 kVa & 8,269 & 11,703 & 11,703 & 12,990 & 12,990 \\
\hline Energy charge/kWh & 53 & 77 & 77 & 85 & 85 \\
\hline Fixed monthly charge & 100,000 & 122,525 & 122,525 & 136,003 & 136,003 \\
\hline Maximum demand ,2001- 75,000 kVa & 16,754 & 17,646 & 17,646 & 19,587 & 19,587 \\
\hline Energy charge/kWh & 39 & 57 & 57 & 63 & 63 \\
\hline Fixed monthly charge & 200,000 & 242,050 & 245,050 & 272,006 & 272,006 \\
\hline Maximum demand, above $75,000 \mathrm{kVa}$ & 16,847 & 17,744 & 17,744 & 19,696 & 19,696 \\
\hline Energy charge/kWh & 33 & 47 & 47 & 52 & 52 \\
\hline Fixed monthly charge & 400,000 & 490,101 & 490,101 & 544,012 & 544,012 \\
\hline \multirow[t]{2}{*}{ Number of consumers } & 263,071 & 277,724 & 303,995 & 310,122 & 297,235 \\
\hline & \multicolumn{5}{|c|}{ (Annual percent change unless otherwise indicated) } \\
\hline Total generated electricity $1 /$ & $\ldots$ & -9.6 & 1.9 & -2.3 & 7.9 \\
\hline Transmission loss in percent of total & 3.2 & 3.5 & 3.1 & 3.2 & 3.6 \\
\hline Domestic consumption & $\ldots$ & 8.5 & 6.4 & 5.9 & -3.4 \\
\hline Number of consumers & $\ldots$ & 5.6 & 9.5 & 2.0 & -4.2 \\
\hline
\end{tabular}

Source: Energy Regulation Board.

1/ Total generated electricity in the interconnecting system and self contained system.

2/ The Copperbelt Energy Corporation purchases from ZESCO and distributes to major mining companies.

$3 /$ This refers to maximum rate. 
Table 12. Zambia: Central Government Operations 1/

\begin{tabular}{|c|c|c|c|c|c|}
\hline & 2002 & 2003 & 2004 & 2005 & 2006 \\
\hline & \multicolumn{5}{|c|}{ (In billions of kwacha) } \\
\hline Revenue and grants & 4,259 & 5,103 & 6,173 & 7,467 & 16,825 \\
\hline Revenue & 2,909 & 3,679 & 4,740 & 5,642 & 6,618 \\
\hline Tax revenue & 2,849 & 3,546 & 4,546 & 5,512 & 6,317 \\
\hline Company income tax & 276 & 246 & 332 & 448 & 695 \\
\hline Personal income tax & 968 & 1,365 & 1,696 & 1,968 & 2,207 \\
\hline Excise taxes & 423 & 482 & 607 & 768 & 821 \\
\hline Value-added tax (VAT) & 812 & 1,034 & 1,362 & 1,633 & 1,792 \\
\hline Trade taxes & 367 & 409 & 544 & 656 & 744 \\
\hline Extraction royalty & 3 & 10 & 4 & 39 & 59 \\
\hline Nontax revenue & 60 & 132 & 194 & 130 & 301 \\
\hline Grants & 1,350 & 1,424 & 1,433 & 1,825 & 10,207 \\
\hline Of which: Debt reduction (including MDRI) & $\ldots$ & $\ldots$ & $\ldots$ & $\ldots$ & 8,410 \\
\hline Total expenditures and net lending & 5,086 & 6,337 & 6,919 & 8,350 & 9,051 \\
\hline Current expenditure & 3,014 & 3,950 & 4,569 & 5,828 & 7,203 \\
\hline Wages and salaries & 1,301 & 1,728 & 2,012 & 2,455 & 2,833 \\
\hline Goods and services & 546 & 612 & 784 & 1,249 & 1,779 \\
\hline Interest payments & 660 & 792 & 898 & 865 & 749 \\
\hline Domestic & 450 & 563 & 746 & 731 & 689 \\
\hline External & 210 & 229 & 152 & 133 & 60 \\
\hline Other & 507 & 817 & 876 & 1,259 & 1,842 \\
\hline Of which: transfers and subsidies & 412 & 362 & 423 & $\ldots$ & 1,526 \\
\hline Capital expenditure & 1,925 & 2,335 & 2,265 & 2,267 & 1,601 \\
\hline Domestic arrears payments & 147 & 52 & 84 & 254 & 247 \\
\hline Savings & -104 & -271 & 171 & -186 & -585 \\
\hline Primary balance after grants & -236 & -542 & 697 & 6 & 8,040 \\
\hline Change in balances and other & -69 & -100 & 545 & 24 & -483 \\
\hline Overall balance (cash) & -896 & $-1,334$ & -201 & -858 & 7,291 \\
\hline Financing & 896 & 1,334 & 201 & 858 & $-7,291$ \\
\hline External financing (net) & 693 & 308 & 230 & 241 & $-6,225$ \\
\hline Budget support & 295 & 46 & 96 & 84 & 36 \\
\hline Project loans & $\ldots$ & $\ldots$ & $\ldots$ & 608 & 298 \\
\hline Amortization & $\ldots$ & $\ldots$ & $\ldots$ & -451 & -167 \\
\hline Debt reduction (MDRI) & $\ldots$ & $\ldots$ & $\ldots$ & $\ldots$ & $-6,392$ \\
\hline Domestic financing (net) & 202 & 1,026 & -29 & 617 & $-1,066$ \\
\hline Bank financing & -45 & 964 & -196 & -96 & $-1,807$ \\
\hline Of which: BOZ onlending of IMF MDRI & $\ldots$ & $\ldots$ & $\ldots$ & $\ldots$ & $-2,018$ \\
\hline Nonbank financing & 247 & 62 & 167 & 713 & 740 \\
\hline
\end{tabular}


Table 12. Zambia: Central Government Operations (concluded) 1/

\begin{tabular}{|c|c|c|c|c|c|}
\hline & 2002 & 2003 & 2004 & 2005 & 2006 \\
\hline & \multicolumn{5}{|c|}{ (In percent of GDP) } \\
\hline Revenue and grants & 26.2 & 24.9 & 23.7 & 23.0 & 42.9 \\
\hline Revenue & 17.9 & 18.0 & 18.2 & 17.4 & 16.9 \\
\hline Tax revenue & 17.5 & 17.3 & 17.5 & 17.0 & 16.1 \\
\hline Company income tax & 1.7 & 1.2 & 1.3 & 1.4 & 1.8 \\
\hline Personal income tax & 6.0 & 6.7 & 6.5 & 6.1 & 5.6 \\
\hline Excise taxes & 2.6 & 2.4 & 2.3 & 2.4 & 2.1 \\
\hline Value-added tax (VAT) & 5.0 & 5.1 & 5.2 & 5.0 & 4.6 \\
\hline Trade taxes & 2.3 & 2.0 & 2.1 & 2.0 & 1.9 \\
\hline Extraction royalty & 0.0 & 0.0 & 0.0 & 0.1 & 0.1 \\
\hline Nontax revenue & 0.4 & 0.6 & 0.7 & 0.4 & 0.8 \\
\hline Grants & 8.3 & 7.0 & 5.5 & 5.6 & 26.0 \\
\hline Total expenditures and net lending & 31.3 & 30.9 & 26.6 & 25.7 & 23.1 \\
\hline Current expenditure & 18.5 & 19.3 & 17.6 & 18.0 & 18.4 \\
\hline Wages and salaries & 8.0 & 8.4 & 7.7 & 7.6 & 7.2 \\
\hline Goods and services & 3.4 & 3.0 & 3.0 & 3.8 & 4.5 \\
\hline Interest payments & 4.1 & 3.9 & 3.5 & 2.7 & 1.9 \\
\hline Domestic & 2.8 & 2.8 & 2.9 & 2.3 & 1.8 \\
\hline External & 1.3 & 1.1 & 0.6 & 0.4 & 0.2 \\
\hline Other & 3.1 & 4.0 & 3.4 & 3.9 & 4.7 \\
\hline Capital expenditure & 11.8 & 11.4 & 8.7 & 7.0 & 4.1 \\
\hline Domestic arrears payments & 0.9 & 0.3 & 0.3 & 0.8 & 0.6 \\
\hline Savings & -0.6 & -1.3 & 0.7 & -0.6 & -1.5 \\
\hline Primary balance after grants & -1.5 & -2.6 & 2.7 & 0.0 & 20.5 \\
\hline Change in balances and other & -0.4 & -0.5 & 2.1 & 0.1 & -1.2 \\
\hline Overall balance (cash) & -5.5 & -6.5 & -0.8 & -2.6 & 18.6 \\
\hline Financing & 5.5 & 6.5 & 0.8 & 2.6 & -18.6 \\
\hline External financing (net) & 4.3 & 1.5 & 0.9 & 0.7 & -15.9 \\
\hline Budget support & 1.8 & 0.2 & 0.4 & 0.3 & 0.1 \\
\hline Project loans & $\ldots$ & $\ldots$ & $\ldots$ & 1.9 & 0.8 \\
\hline Amortization & $\ldots$ & $\ldots$ & $\ldots$ & -1.4 & -0.4 \\
\hline Debt reduction (MDRI) & $\ldots$ & $\ldots$ & $\ldots$ & $\ldots$ & -16.3 \\
\hline Domestic financing (net) & 1.2 & 5.0 & -0.1 & 1.9 & -2.7 \\
\hline
\end{tabular}

Sources: Ministry of Finance and National Planning.

1/ This excludes extra-budgetary transactions. 
Table 13. Zambia: National Pension Scheme Authority Operations 1/

\begin{tabular}{|c|c|c|c|c|c|}
\hline & 2002 & 2003 & 2004 & 2005 & 2006 \\
\hline & \multicolumn{5}{|c|}{ (In billions of kwacha unless otherwise indicated) } \\
\hline Total income & 186.0 & 276.0 & 300.0 & 296.0 & 465.0 \\
\hline Contributions & 99.0 & 145.0 & 184.0 & 241.0 & 292.0 \\
\hline Investment & 84.0 & 125.0 & 107.0 & 122.0 & 141.0 \\
\hline Other & 3.0 & 6.0 & 9.0 & -67.0 & 32.0 \\
\hline Total expenditure & 61.6 & 68.2 & 82.1 & 98.2 & 96.1 \\
\hline Administrative expenditure & 42.0 & 46.0 & 47.0 & 59.0 & 57.0 \\
\hline Benefits & 13.6 & 21.2 & 32.1 & 35.2 & 35.1 \\
\hline Maternity benefit 2/ & 0.1 & 0.0 & 0.0 & 0.0 & 0.0 \\
\hline Old-age benefit & 3.8 & 6.5 & 9.9 & 10.5 & 11.6 \\
\hline Invalidity benefit & 4.2 & 3.0 & 4.7 & 7.4 & 8.7 \\
\hline Survivors benefit & 1.2 & 4.7 & 11.1 & 8.6 & 9.5 \\
\hline Funeral benefit & 0.1 & 0.1 & 0.1 & 0.1 & 0.1 \\
\hline Homeownership 2/ & 4.3 & 6.9 & 6.4 & 8.6 & 5.2 \\
\hline Capital expenditure & 6.0 & 1.0 & 3.0 & 4.0 & 4.0 \\
\hline Overall balance & 124.4 & 207.8 & 217.9 & 197.8 & 368.9 \\
\hline Of which: In percent of GDP & 0.8 & 1.0 & 0.8 & 0.6 & 0.9 \\
\hline Accumulated funds (accrual) & 373.0 & 600.0 & 894.0 & 1108.0 & 1494.0 \\
\hline Of which: In percent of GDP & 2.3 & 2.9 & 3.4 & 3.4 & 3.8 \\
\hline \multicolumn{6}{|l|}{ Memorandum items: } \\
\hline Accumulated funds to expenditure ratio & 6.1 & 8.8 & 10.9 & 11.3 & 15.5 \\
\hline Investment returns to accumulated funds & 22.5 & 20.8 & 12.0 & 11.0 & 9.4 \\
\hline Contribution rate, end of period, in percent & 10.0 & 10.0 & 10.0 & 10.0 & 10.0 \\
\hline Employer & 5.0 & 5.0 & 5.0 & 5.0 & 5.0 \\
\hline Employee & 5.0 & 5.0 & 5.0 & 5.0 & 5.0 \\
\hline Number of contributors $3 /$ & 320,125 & 392,035 & 356,089 & 492,101 & 449,167 \\
\hline Annual change & -12.1 & 22.5 & -9.2 & 38.2 & -8.7 \\
\hline Average insurable wages & 345,514 & 390,690 & 441,773 & 518,451 & 809,375 \\
\hline Annual change & 32.8 & 13.1 & 13.1 & 17.4 & 56.1 \\
\hline
\end{tabular}

Source: National Pension Scheme Authority.

$1 /$ This is a consolidation of the operations under the Zambia National Provident Fund (ZNPF) and the National Pension Scheme Act of 1996 (NAPSA). The NAPSA began operations in February 2000 and manages the assets and liabilities of members who contributed to a defined contribution plan, ZNPF.

2/ These transfers are to ZNPF members.

$3 /$ This refers to NAPSA active contributing members that includes seasonal workers. 
Table 14. Zambia: Monetary Survey

\begin{tabular}{|c|c|c|c|c|c|}
\hline & 2002 & 2003 & 2004 & 2005 & 2006 \\
\hline & \multicolumn{5}{|c|}{ (In billions of kwacha; end of period) } \\
\hline Net foreign assets & $-3,159$ & $-2,435$ & $-1,969$ & -282 & 3,953 \\
\hline Monetary authorities & $-4,332$ & $-3,413$ & $-3,376$ & $-1,023$ & 2,807 \\
\hline Commercial banks & 1,173 & 978 & 1,407 & 741 & 1,146 \\
\hline Net domestic assets & 6,779 & 6,903 & 7,789 & 6,123 & 4,524 \\
\hline Net domestic credit & 2,857 & 4,215 & 4,766 & 5,054 & 6,358 \\
\hline \multirow{2}{*}{$\begin{array}{l}\text { Net claims on general government } 1 / \\
\text { Of which: net claims of central government }\end{array}$} & 1,744 & 2,709 & 2,513 & 2,387 & 2,374 \\
\hline & 1,744 & 2,709 & 2,513 & 2,416 & 2,440 \\
\hline Claims on nongovernment & 1,113 & 1,506 & 2,254 & 2,667 & 3,984 \\
\hline Public enterprises & 94 & 116 & 200 & 230 & 224 \\
\hline Private sector & 1,019 & 1,390 & 2,054 & 2,437 & 3,760 \\
\hline Other items (net) & 3,921 & 2,688 & 3,023 & 1,068 & $-1,834$ \\
\hline Broad money & 3,620 & 4,468 & 5,820 & 5,841 & 8,477 \\
\hline Narrow money & 1,334 & 1,701 & 2,052 & 2,289 & 3,473 \\
\hline Currency in circulation & 433 & 600 & 742 & 834 & 1,081 \\
\hline Demand deposits & 902 & 1,101 & 1,310 & 1,455 & 2,392 \\
\hline Quasi money & 2,285 & 2,766 & 3,768 & 3,552 & 5,004 \\
\hline Savings deposits & 465 & 673 & 839 & 898 & 1,087 \\
\hline Time deposits & 392 & 474 & 489 & 672 & 1,203 \\
\hline \multirow[t]{2}{*}{ Foreign currency deposits } & 1,429 & 1,619 & 2,440 & 1,981 & 2,714 \\
\hline & \multicolumn{5}{|c|}{ (12-month percent change) } \\
\hline Net foreign assets & 11.1 & -22.9 & -19.1 & -85.7 & $-1,503.8$ \\
\hline Net domestic assets & 21.1 & 1.8 & 12.8 & -21.4 & -26.1 \\
\hline Net domestic credit & 10.2 & 47.5 & 13.1 & 6.0 & 25.8 \\
\hline Net claims on general government $1 /$ & -5.6 & 55.3 & -7.2 & -5.0 & -0.5 \\
\hline Claims on nongovernment & -19.8 & 35.3 & 49.6 & 18.4 & 49.4 \\
\hline Other items (net) & 30.6 & -31.5 & 12.5 & -64.7 & -271.7 \\
\hline Broad money & 31.4 & 23.4 & 30.3 & 0.4 & 45.1 \\
\hline Narrow money & 31.5 & 27.5 & 20.6 & 11.6 & 51.7 \\
\hline \multirow[t]{2}{*}{ Quasi money } & 31.5 & 21.1 & 36.2 & -5.7 & 40.9 \\
\hline & \multicolumn{5}{|c|}{ (Percent contribution to growth in broad money) } \\
\hline Net foreign assets & -11.5 & 20.0 & 10.4 & 29.0 & 72.5 \\
\hline Net domestic assets & 43.0 & 3.4 & 19.8 & -28.6 & -27.4 \\
\hline Net domestic credit & 9.6 & 37.5 & 12.3 & 4.9 & 22.3 \\
\hline Net claims on general government $1 /$ & -3.8 & 26.6 & -4.4 & -2.2 & -0.2 \\
\hline Claims on nongovernment & -10.0 & 10.9 & 16.7 & 7.1 & 22.5 \\
\hline Other items (net) & 33.4 & -34.1 & 7.5 & -33.6 & -49.7 \\
\hline Broad money & 31.4 & 23.4 & 30.3 & 0.4 & 45.1 \\
\hline Narrow money & 11.6 & 10.1 & 7.8 & 4.1 & 20.3 \\
\hline Quasi money & 19.9 & 13.3 & 22.4 & -3.7 & 24.9 \\
\hline \multicolumn{6}{|l|}{ Memorandum items: } \\
\hline Velocity (GDP/average M2) & 5.10 & 5.06 & 5.05 & 5.57 & 5.48 \\
\hline Money multiplier 2/ & 2.9 & 3.1 & 3.1 & 2.4 & 2.6 \\
\hline
\end{tabular}

Sources: Bank of Zambia.

1/ Includes debt stock reduction under the IMF's Multilateral Debt Relief Initiative, SDR 402.6 million in 2006.

2/ Ratio of broad money to reserve money. 
Table 15. Zambia: Summary Accounts of the Bank of Zambia

(In billions of kwacha; end of period)

\begin{tabular}{|c|c|c|c|c|c|}
\hline & 2002 & 2003 & 2004 & 2005 & 2006 \\
\hline Net foreign assets & $-4,332.2$ & $-3,413.2$ & $-3,376.4$ & $-1,023.0$ & $2,807.2$ \\
\hline Foreign assets & $4,271.4$ & $4,634.8$ & $4,852.1$ & $4,751.3$ & $5,334.1$ \\
\hline Of which: foreign reserve assets & $4,256.0$ & $4,628.9$ & $4,529.3$ & $4,565.5$ & $5,331.7$ \\
\hline Foreign liabilities $1 /$ & $-8,603.6$ & $-8,048.0$ & $-8,228.5$ & $-5,774.3$ & $-2,527.0$ \\
\hline Of which: Liabilities to IMF & $-7,816.6$ & $-7,334.8$ & $-7,960.0$ & $-5,637.1$ & $-2,457.3$ \\
\hline Net domestic assets & $5,597.1$ & $4,857.6$ & $5,270.4$ & $3,430.9$ & 419.7 \\
\hline Domestic credit & $1,152.0$ & $1,436.6$ & $1,610.2$ & $1,444.8$ & $1,153.7$ \\
\hline Claims on general government (net) $1 /$ & 958.4 & $1,073.7$ & $1,280.5$ & $1,070.3$ & 844.3 \\
\hline Of which: Claims on central government & 958.4 & $1,073.7$ & $2,172.0$ & $1,939.7$ & $1,945.0$ \\
\hline Claims on public enterprises & 30.5 & 54.3 & 85.5 & 96.3 & 0.0 \\
\hline Claims on Deposit money banks & 112.2 & 261.6 & 203.8 & 225.6 & 261.9 \\
\hline Claims on private sector & 50.9 & 46.9 & 40.3 & 52.6 & 47.4 \\
\hline Other items (net) & $4,445.1$ & $3,421.0$ & $3,660.2$ & $1,986.1$ & -734.0 \\
\hline Of which: valuation & 66.0 & 14.6 & 170.6 & 401.5 & 766.5 \\
\hline Reserve money (inclusive of OMO deposits) & $1,264.9$ & $1,444.4$ & $1,894.0$ & $2,407.9$ & $3,226.9$ \\
\hline Currency outside banks and cash in vaults & 479.4 & 670.1 & 818.5 & 962.0 & $1,224.2$ \\
\hline Liabilities to commercial banks & 774.9 & 766.4 & $1,066.0$ & $1,434.7$ & $1,992.7$ \\
\hline Liabilities to nonbanks & 10.5 & 7.8 & 9.5 & 11.2 & 10.0 \\
\hline
\end{tabular}

Source: Bank of Zambia.

1/ Includes debt stock reduction under the IMF's Multilateral Debt Relief Initiative, SDR 402.6 million in 2006. 
Table 16. Zambia: Accounts of Commercial Banks

(In billions of kwacha)

\begin{tabular}{|c|c|c|c|c|c|}
\hline & 2002 & 2003 & 2004 & 2005 & 2006 \\
\hline Net foreign assets & $1,173.1$ & 978.5 & $1,407.0$ & 741.4 & $1,146.0$ \\
\hline Gross assets & $1,272.1$ & $1,093.2$ & $1,593.8$ & $1,205.3$ & $1,838.5$ \\
\hline Liabilities & -99.1 & -114.7 & -186.8 & -463.9 & -692.5 \\
\hline Net domestic assets & $2,013.8$ & $2,889.1$ & $3,670.8$ & $4,265.4$ & $6,249.7$ \\
\hline Reserves (Credit to BOZ) & 731.3 & 766.0 & $1,108.7$ & $1,483.9$ & $2,242.6$ \\
\hline Cash in vaults & 57.1 & 77.7 & 85.9 & 138.8 & 153.0 \\
\hline Other balances at $\mathrm{BOZ}$ & 119.7 & 198.6 & 242.6 & 260.8 & 388.5 \\
\hline Statutory reserves at $\mathrm{BOZ}$ (kwacha and forex) & 492.7 & 489.8 & 643.4 & 715.1 & 992.6 \\
\hline Money market placements & 61.8 & 0.0 & 136.8 & 369.2 & 708.5 \\
\hline Net domestic credit & $1,817.7$ & $3,040.1$ & $3,359.9$ & $3,835.1$ & $5,466.3$ \\
\hline Claims on general government (net) & 786.1 & $1,635.1$ & $1,232.2$ & $1,316.6$ & $1,529.7$ \\
\hline Claims on general government & 916.3 & $1,858.1$ & $1,707.9$ & $1,834.3$ & $2,063.0$ \\
\hline Treasury bills & 516.3 & $1,091.3$ & 865.4 & 913.3 & $1,066.8$ \\
\hline Other assets & 400.1 & 766.9 & 842.6 & 920.9 & 996.2 \\
\hline Deposits of general government & -130.3 & -223.0 & -475.8 & -517.6 & -533.3 \\
\hline Claims on parastatals \& state enterpr. & 63.0 & 61.8 & 114.6 & 134.0 & 224.4 \\
\hline Claims on private enterprises & 830.6 & $1,078.9$ & $1,735.4$ & $1,865.6$ & $3,038.6$ \\
\hline Claims on households & 126.4 & 245.5 & 275.8 & 489.0 & 583.7 \\
\hline Claims on nonbank fin. inst. & 11.6 & 18.9 & 1.9 & 29.9 & 89.9 \\
\hline Other items net & -535.2 & -917.0 & -797.8 & $-1,053.6$ & $-1,459.2$ \\
\hline Assets & 947.2 & 525.6 & 743.6 & 988.9 & 977.3 \\
\hline Liabilities & $-1,482.4$ & $-1,442.6$ & $-1,541.5$ & $-2,042.6$ & $-2,436.5$ \\
\hline Liabilities to nongovernment sector & $3,186.8$ & $3,867.6$ & $5,077.8$ & $5,006.8$ & $7,395.7$ \\
\hline Demand deposits in Kwacha & 896.7 & $1,101.2$ & $1,309.5$ & $1,454.7$ & $2,391.2$ \\
\hline Demand deposits in forex & $1,332.6$ & $1,537.6$ & $2,098.8$ & $1,688.4$ & $2,502.0$ \\
\hline Savings deposits in Kwacha & 464.7 & 673.2 & 839.4 & 898.4 & $1,086.6$ \\
\hline Savings deposits in forex & 11.6 & 15.0 & 15.8 & 19.9 & 12.9 \\
\hline Time deposits in Kwacha & 391.6 & 474.2 & 489.2 & 672.4 & $1,203.4$ \\
\hline Time deposits in forex & 84.8 & 66.6 & 325.0 & 273.0 & 199.1 \\
\hline Bills payable & 4.8 & 0.0 & 0.1 & 0.2 & 0.4 \\
\hline
\end{tabular}

Source: Bank of Zambia. 
Table 17. Zambia: Net Foreign Assets of the Banking System (In billions of kwacha)

\begin{tabular}{lrrrrr}
\hline & 2002 & 2003 & 2004 & 2005 & 2006 \\
& & & & & \\
\hline & & & & & \\
Net foreign assets of the banking system & $-3,159$ & $-2,435$ & $-1,969$ & -282 & 3,953 \\
$\quad$ Assets & 5,544 & 5,728 & 6,446 & 5,957 & 7,173 \\
$\quad$ Liabilities & $-8,703$ & $-8,163$ & $-8,415$ & $-6,238$ & $-3,220$ \\
& & & & & \\
Net foreign assets of the Bank of Zambia & $-4,332$ & $-3,413$ & $-3,376$ & $-1,023$ & 2,807 \\
$\quad$ Assets & 4,271 & 4,635 & 4,852 & 4,751 & 5,334 \\
$\quad$ Liabilities 1/ & $-8,604$ & $-8,048$ & $-8,229$ & $-5,774$ & $-2,527$ \\
$\quad$ IMF 1/ & $-7,817$ & $-7,335$ & $-7,960$ & $-5,637$ & $-2,457$ \\
$\quad$ Other & -787 & -713 & -269 & -137 & -70 \\
& & & & & \\
Net foreign assets of commercial banks & 1,173 & 978 & 1,407 & 741 & 1,146 \\
$\quad$ Assets & 1,272 & 1,093 & 1,594 & 1,205 & 1,839 \\
$\quad$ Liabilities & -99 & -115 & -187 & -464 & -693 \\
& & & & & \\
\hline
\end{tabular}

Sources: Bank of Zambia.

1/ Includes debt stock reduction under the IMF's Multilateral Debt Relief Initiative, SDR 402.6 million in 2006. 
Table 18. Zambia: Commercial Bank Loans and Advances by Sector

\begin{tabular}{|c|c|c|c|c|c|}
\hline & 2002 & 2003 & 2004 & 2005 & 2006 \\
\hline & \multicolumn{5}{|c|}{ (In millions of Zambia kwacha) } \\
\hline Credit to nongovernment sector & 1113.0 & 1506.3 & 2253.7 & 2667.5 & 3984.0 \\
\hline Agriculture, forestry, and fishing & 228 & 346 & 637 & 679 & 1066 \\
\hline Mining and quarrying & 37 & 68 & 94 & 97 & 171 \\
\hline Manufacturing & 144 & 178 & 274 & 304 & 520 \\
\hline Energy and water & 19 & 44 & 131 & 145 & 124 \\
\hline Construction & 17 & 30 & 42 & 49 & 87 \\
\hline Wholesale and retail trade & 187 & 202 & 292 & 357 & 568 \\
\hline Restaurants and hotels & 51 & 52 & 76 & 58 & 78 \\
\hline Transport, storage, and communications & 60 & 101 & 161 & 187 & 312 \\
\hline Financial services & 15 & 29 & 22 & 61 & 186 \\
\hline Community, social, and personal services & 21 & 22 & 71 & 58 & 98 \\
\hline Real estate & 6 & 9 & 52 & 76 & 190 \\
\hline Personal loans & 240 & 320 & 217 & 354 & 438 \\
\hline \multirow[t]{2}{*}{ Other } & 86.9 & 105.3 & 183.5 & 240.7 & 145.0 \\
\hline & \multicolumn{5}{|c|}{ (In percent of total) } \\
\hline Agriculture, forestry, and fishing & 20.5 & 22.9 & 28.3 & 25.5 & 26.8 \\
\hline Mining and quarrying & 3.4 & 4.5 & 4.2 & 3.6 & 4.3 \\
\hline Manufacturing & 12.9 & 11.8 & 12.2 & 11.4 & 13.1 \\
\hline Energy and water & 1.7 & 2.9 & 5.8 & 5.4 & 3.1 \\
\hline Construction & 1.5 & 2.0 & 1.9 & 1.9 & 2.2 \\
\hline Wholesale and retail trade & 16.8 & 13.4 & 13.0 & 13.4 & 14.3 \\
\hline Restaurants and hotels & 4.6 & 3.4 & 3.4 & 2.2 & 2.0 \\
\hline Transport, storage, and communications & 5.4 & 6.7 & 7.1 & 7.0 & 7.8 \\
\hline Financial services & 1.4 & 1.9 & 1.0 & 2.3 & 4.7 \\
\hline Community, social, and personal services & 1.9 & 1.5 & 3.2 & 2.2 & 2.5 \\
\hline Real estate & 0.6 & 0.6 & 2.3 & 2.8 & 4.8 \\
\hline Personal loans & 21.5 & 21.2 & 9.6 & 13.3 & 11.0 \\
\hline \multirow[t]{2}{*}{ Other } & 7.8 & 7.0 & 8.1 & 9.0 & 3.6 \\
\hline & \multicolumn{5}{|c|}{ (Contribution to credit to nongovernment sector) } \\
\hline Credit to nongovernment sector & -19.8 & 35.3 & 49.6 & 18.4 & 49.4 \\
\hline Agriculture, forestry, and fishing & 0.5 & 10.6 & 19.4 & 1.9 & 14.5 \\
\hline Mining and quarrying & -4.7 & 2.8 & 1.7 & 0.1 & 2.8 \\
\hline Manufacturing & 1.8 & 3.1 & 6.4 & 1.4 & 8.1 \\
\hline Energy and water & -14.9 & 2.3 & 5.8 & 0.6 & -0.8 \\
\hline Construction & 0.3 & 1.2 & 0.8 & 0.3 & 1.4 \\
\hline Wholesale and retail trade & 5.4 & 1.3 & 6.0 & 2.9 & 7.9 \\
\hline Restaurants and hotels & 0.2 & 0.0 & 1.6 & -0.8 & 0.7 \\
\hline Transport, storage, and communications & 1.6 & 3.7 & 4.0 & 1.2 & 4.7 \\
\hline Financial services & 0.5 & 1.2 & -0.4 & 1.7 & 4.7 \\
\hline Community, social, and personal services & 0.6 & 0.1 & 3.3 & -0.6 & 1.5 \\
\hline Real estate & 0.1 & 0.3 & 2.9 & 1.0 & 4.3 \\
\hline Personal loans & 1.4 & 7.2 & -6.8 & 6.1 & 3.2 \\
\hline Other & -12.5 & 1.7 & 5.2 & 2.5 & -3.6 \\
\hline
\end{tabular}

Source: Bank of Zambia. 
Table 19. Zambia: Structure of Interest Rates

(In percent; end of period)

\begin{tabular}{|c|c|c|c|c|c|}
\hline & 2002 & 2003 & 2004 & 2005 & 2006 \\
\hline Bank rate & 34.5 & 15.8 & 18.3 & 17.1 & 10.7 \\
\hline Treasury bill rate $1 /$ & 31.7 & 19.7 & 18.2 & 16.4 & 9.2 \\
\hline 91 days treasury bill rate & 32.5 & 13.8 & 16.3 & 15.1 & 8.7 \\
\hline 182 days treasury bill rate & $\ldots$ & $\ldots$ & 18.3 & 16.3 & 8.7 \\
\hline 273 days treasury bill rate & $\ldots$ & $\ldots$ & 19.4 & 17.0 & 9.6 \\
\hline 364 days treasury bill rate & $\ldots$ & $\ldots$ & $\ldots$ & 17.0 & 9.9 \\
\hline 12-month government bond & 42.7 & 22.0 & 19.6 & 16.0 & $\ldots$ \\
\hline 18-month government bond & 43.3 & 25.2 & 20.8 & 17.0 & $\ldots$ \\
\hline 24-month government bond & 43.5 & 26.1 & 22.0 & 19.0 & 10.6 \\
\hline 3-year government bond & $\ldots$ & $\ldots$ & $\ldots$ & 22.3 & 12.1 \\
\hline 5-year government bond & $\ldots$ & $\ldots$ & $\ldots$ & 25.0 & 13.5 \\
\hline \multicolumn{6}{|l|}{ Kwacha deposit rates } \\
\hline 3 months & 22.5 & 21.1 & 11.1 & 10.4 & 10.3 \\
\hline 6 months & 22.3 & 20.4 & 10.9 & 9.5 & 9.4 \\
\hline 24-hour call rate & 7.9 & 8.1 & 5.3 & 4.6 & 4.9 \\
\hline \multicolumn{6}{|l|}{ Lending rates } \\
\hline Weighted interbank rate & $\ldots$ & 5.5 & 14.4 & 20.6 & 7.4 \\
\hline Weighted lending base rate & $\ldots$ & 37.7 & 29.8 & 27.6 & 21.6 \\
\hline
\end{tabular}

Source: Bank of Zambia.

1/ Annualized (weighted by maturity). 
Table 20. Zambia: Financial Soundness Indicators

\begin{tabular}{|c|c|c|c|c|c|}
\hline & 2002 & 2003 & 2004 & 2005 & 2006 \\
\hline \multicolumn{6}{|c|}{ (In percent, unless otherwise indicated) } \\
\hline \multicolumn{6}{|l|}{ Capital adequacy } \\
\hline Regulatory capital to risk-weighted assets & 28.0 & 23.0 & 22.2 & 28.4 & 20.4 \\
\hline Tier 1 regulatory capital to risk-weighted asse & 24.8 & 21.0 & 19.6 & 26.2 & 18 \\
\hline Capital to total assets & 12.3 & 11.0 & 9.7 & 11.6 & 9.1 \\
\hline \multicolumn{6}{|l|}{ Asset quality } \\
\hline Past due advances (NPL) to total advances & 11.4 & 5.3 & 7.6 & 8.9 & 11.3 \\
\hline Loan loss provisions to nonperforming loans & 73.9 & 89.3 & 102.8 & 90.7 & 83.3 \\
\hline Bad debt provisions to advances & 8.4 & 4.7 & 7.8 & 5.4 & 6.8 \\
\hline \multicolumn{6}{|l|}{ Loan concentration 1/ } \\
\hline Households & 12.3 & 17.5 & 13.1 & 19.5 & 16.1 \\
\hline Government and parastatals & 6.4 & 4.3 & 5.5 & 5.5 & 6.2 \\
\hline Agriculture & 22.2 & 24.7 & 30.3 & 27.0 & 23.9 \\
\hline Mining & 3.6 & 4.9 & 4.5 & 4.0 & 4.4 \\
\hline Manufacturing & 14.0 & 12.7 & 13.0 & 12.0 & 14 \\
\hline Construction & 1.6 & 2.2 & 2.0 & 2.0 & 2.4 \\
\hline Services & 8.5 & 7.3 & 8.1 & 7.0 & 9.8 \\
\hline Others & 49.9 & 45.1 & 42.0 & 48.0 & 45.5 \\
\hline \multicolumn{6}{|l|}{ Earnings and profitability } \\
\hline Return on average assets & 6.5 & 5.4 & 3.1 & 6.5 & 5.1 \\
\hline Return on equity & 52.8 & 48.5 & 29.8 & 46.4 & 30.6 \\
\hline Gross interest income to total gross income & 65.8 & 66.1 & 78.2 & 60.9 & 60.2 \\
\hline Gross noninterest income to total gross incon & 34.2 & 33.9 & 46.3 & 39.1 & 39.8 \\
\hline Net interest margin & 15.3 & 13.2 & 11.8 & 11.8 & 12.8 \\
\hline \multicolumn{6}{|l|}{ Liquidity $2 /$} \\
\hline Liquid assets to total assets & 78.6 & 74.7 & 66.6 & 41.0 & 41.3 \\
\hline Liquid assets to total deposits & 69.7 & 73.5 & 73.7 & 51.0 & 49.6 \\
\hline Advances to deposits ratio & 29.9 & 33.3 & 37.3 & 44.5 & 49 \\
\hline \multicolumn{6}{|l|}{ Exposure to foreign currency } \\
\hline Foreign currency loans to total gross loans & 42.8 & 46.8 & 41.2 & 36.2 & 34.0 \\
\hline Foreign currency liabilities to total liabilities & 62.2 & 58.4 & 58.4 & 31.0 & 61.2 \\
\hline Net open position in foreign exchange to capi & 15.7 & 10.0 & 10.0 & 2.1 & 9.4 \\
\hline \multicolumn{6}{|l|}{ Exposure to household debt } \\
\hline Household debt to GDP & 0.0 & 0.0 & 0.0 & 1.5 & 1.2 \\
\hline
\end{tabular}

Source: Bank of Zambia.

1/ Components do not add up to 100 because loans to households, government, and parastatals are included in loans classified by economic sectors.

2/ Liquid assets were redefined to exclude one-year Treasury bills beginning in 2005. 
Table 21. Zambia: Balance of Payments

(In millions of U.S. dollars)

\begin{tabular}{|c|c|c|c|c|c|}
\hline & \multicolumn{5}{|c|}{ Preliminary } \\
\hline & 2002 & 2003 & 2004 & 2005 & 2006 \\
\hline Current Account & -579 & -641 & -618 & -662 & 120 \\
\hline Merchandise trade balance & -259 & -302 & -25 & 50 & 1,183 \\
\hline Exports, f.o.b & 944 & 1,090 & 1,847 & 2,210 & 3,819 \\
\hline Imports, f.o.b & $-1,204$ & $-1,393$ & $-1,872$ & $-2,161$ & $-2,636$ \\
\hline Services (net) & -245 & -238 & -215 & -199 & -284 \\
\hline Services Receipts & 136 & 165 & 232 & 272 & 303 \\
\hline Services Payments & -381 & -403 & -447 & -471 & -587 \\
\hline Income Net & -151 & -143 & -397 & -619 & -960 \\
\hline Of which: Official interest payments & -132 & -126 & -121 & -110 & -17 \\
\hline Current Transfers (net) & 76 & 42 & 19 & 107 & 180 \\
\hline Of which: General Government & 96 & 65 & 44 & 131 & 260 \\
\hline Capital and Financial Account & 387 & 421 & 258 & 704 & 1,013 \\
\hline Capital Account & 236 & 240 & 239 & 287 & 2,600 \\
\hline General Government & 236 & 240 & 239 & 287 & 2,600 \\
\hline Debt Forgiveness / 1 & 0 & 0 & 0 & 0 & 2,403 \\
\hline Project Assistance grants & 236 & 240 & 239 & 287 & 197 \\
\hline Other Sectors & 0 & 0 & 0 & 0 & 0 \\
\hline Financial Account & 151 & 181 & 19 & 417 & $-1,587$ \\
\hline Central government (net) & -53 & -131 & -201 & -65 & $-1,779$ \\
\hline Disbursements & 181 & 111 & 131 & 160 & 91 \\
\hline Amortization 1/ & -234 & -242 & -331 & -225 & $-1,870$ \\
\hline Nongovernment capital (net) & 204 & 312 & 220 & 482 & 192 \\
\hline Foreign direct investment (net) & 303 & 347 & 364 & 380 & 467 \\
\hline Change in NFA of banks (- increase) & -53 & 48 & -90 & 91 & 62 \\
\hline Other (net) & -47 & -83 & -54 & 11 & -337 \\
\hline Errors and Omissions & -20 & -8 & 139 & -172 & -311 \\
\hline Overall balance & -211 & -228 & -220 & -129 & 822 \\
\hline
\end{tabular}


Table 21. Zambia: Balance of Payments (concluded)

(In millions of U.S. dollars)

\begin{tabular}{|c|c|c|c|c|c|}
\hline & \multicolumn{5}{|c|}{ Preliminary } \\
\hline & 2002 & 2003 & 2004 & 2005 & 2006 \\
\hline Overall balance & -211 & -228 & -220 & -129 & 822 \\
\hline Financing & 212 & 228 & 220 & 129 & -821 \\
\hline Change in net int. reserves of BoZ (-increase) & -225 & -161 & -44 & -351 & -821 \\
\hline Gross official reserves of BoZ (-increase) & -169 & 89 & -28 & -109 & -264 \\
\hline BoZ liabilities & -6 & -6 & -6 & -6 & 0 \\
\hline IMF (net) & -50 & -244 & -10 & -236 & -557 \\
\hline Disbursements & 173 & 0 & 248 & 16 & 24 \\
\hline Repayments & -222 & -244 & -257 & -252 & -582 \\
\hline Exceptional financing & 437 & 389 & 264 & 480 & 0 \\
\hline Non-HIPC & 171 & 154 & 245 & 152 & 0 \\
\hline HIPC, including IMF & 266 & 235 & 19 & 328 & 0 \\
\hline \multicolumn{6}{|l|}{ Of which: } \\
\hline IMF & 153 & 169 & 0 & 219 & 0 \\
\hline Paris Club & 64 & 16 & -2 & 38 & 0 \\
\hline \multicolumn{6}{|l|}{ Memorandum items: } \\
\hline Exports of goods and nonfactor services & 1,080 & 1,255 & 2,079 & 2,483 & 4,122 \\
\hline Imports of goods and nonfactor services & $-1,585$ & $-1,796$ & $-2,319$ & $-2,632$ & $-3,223$ \\
\hline Nominal GDP at market prices in US dollars & 3,775 & 4,326 & 5,440 & 7,271 & 10,893 \\
\hline Exchange rate, average (K/US\$) & 4,307 & 4,734 & 4,779 & 4,463 & 3,601 \\
\hline
\end{tabular}

Source: Bank of Zambia and Fund staff estimates.

1/ Includes debt stock reduction under the IMF's Multilateral Debt Relief Initiative, SDR 402.6 million in 2006. 
Table 22. Zambia: Merchandise Exports

\begin{tabular}{|c|c|c|c|c|c|}
\hline & 2002 & 2003 & 2004 & 2005 & 2006 \\
\hline & \multicolumn{5}{|c|}{ (In millions of U.S. dollars, unless otherwise indicated) } \\
\hline Total exports, f.o.b. & 944 & 1,090 & 1,847 & 2,210 & 3,819 \\
\hline Metal exports, f.o.b. & 560 & 669 & 1,359 & 1,644 & 3,084 \\
\hline \multicolumn{6}{|l|}{ Copper } \\
\hline Value & 510 & 607 & 1,075 & 1,486 & 2,938 \\
\hline Volume (thousands of metric tons) & 330.0 & 353.4 & 410.9 & 438.8 & 476.1 \\
\hline Unit value $1 /$ & 1.5 & 1.7 & 2.6 & 3.4 & 6.2 \\
\hline \multicolumn{6}{|l|}{ Cobalt } \\
\hline Value & 50 & 62 & 284 & 158 & 146 \\
\hline Volume (thousands metric tons) & 4.2 & 3.4 & 6.1 & 5.4 & 4.7 \\
\hline Unit value $1 /$ & 11.9 & 18.4 & 46.6 & 29.1 & 31.3 \\
\hline Nontraditional exports, f.o.b. & 360.7 & 399.6 & 468.3 & 534.3 & 701.5 \\
\hline \multirow[t]{2}{*}{ Goods Procured in ports by carriers } & 23.9 & 21.5 & 19.8 & 32.0 & 33.6 \\
\hline & \multicolumn{5}{|c|}{ (In percent of total exports) } \\
\hline Metal exports, f.o.b. & 59.3 & 61.4 & 73.6 & 74.4 & 80.8 \\
\hline Copper & 54.0 & 55.7 & 58.2 & 67.2 & 76.9 \\
\hline Cobalt & 5.3 & 5.7 & 15.4 & 7.2 & 3.8 \\
\hline Nontraditional exports, f.o.b. & 38.2 & 36.7 & 25.4 & 24.2 & 18.4 \\
\hline Goods Procured in ports by carriers & 2.5 & 2.0 & 1.1 & 1.4 & 0.9 \\
\hline
\end{tabular}

Source: Bank of Zambia.

1/ Net of freight and insurance. 
Table 23. Zambia: Foreign Trade Volume and Unit Value (Annual percent change unless otherwise indicated)

\begin{tabular}{lrrrrr}
\hline & 2002 & 2003 & 2004 & 2005 & 2006 \\
\hline Terms of trade (1992=100) & -1.9 & 1.4 & 34.6 & 6.8 & 54.8 \\
& & & & & \\
Export value & 3.6 & 15.8 & 71.1 & 19.9 & 73.8 \\
Export volume & 5.1 & 2.3 & 14.3 & 7.1 & 5.9 \\
Export unit value 1/ & -1.4 & 13.2 & 49.7 & 12.0 & 64.0 \\
& & & & & \\
Exports of metal & & & & & \\
$\quad$ Value & -5.1 & 19.6 & 103.1 & 21.0 & 87.6 \\
$\quad$ Volume & 7.5 & 4.4 & 19.5 & 6.8 & 7.1 \\
Unit values 1/ & -11.7 & 14.5 & 70.0 & 13.3 & 75.2 \\
& & & & & \\
Exports of nonmetal & & & & & \\
$\quad$ Value & 21.1 & 10.0 & 16.6 & 16.9 & 31.3 \\
Volume & -0.9 & -3.5 & -1.0 & 8.1 & 1.9 \\
Unit values 1/ & 22.2 & 13.9 & 17.8 & 8.1 & 28.8 \\
Import value & & & & & \\
Import volume & -3.9 & 15.7 & 34.4 & 15.4 & 22.0 \\
Import unit value 1/ & -4.5 & 3.6 & 20.8 & 10.1 & 15.1 \\
& 0.5 & 11.7 & 11.3 & 4.8 & 6.0 \\
\hline Sourc: Bank of Zambia & & & & & \\
\hline
\end{tabular}

Source: Bank of Zambia.

1/ In U.S. dollar terms. 
Table 24. Zambia: Merchandise Exports by SITC

\begin{tabular}{|c|c|c|c|c|c|}
\hline & 2002 & 2003 & 2004 & 2005 & 2006 \\
\hline & \multicolumn{5}{|c|}{ (In million of U.S. dollars) } \\
\hline Exports, fob & 944.3 & $1,090.4$ & $1,846.9$ & $2,210.4$ & $3,819.2$ \\
\hline Food and live animals & 69.6 & 75.0 & 152.1 & 172.9 & 139.4 \\
\hline Beverages and tobacco & 16.3 & 22.3 & 61.1 & 74.1 & 74.9 \\
\hline Crude materials, (excl fuels) & 72.9 & 91.5 & 237.6 & 258.4 & 536.8 \\
\hline Mineral fuels, lubricants and related materials & 7.8 & 13.7 & 27.8 & 13.9 & 22.6 \\
\hline Animal and vegetable oils, fats and waxes & 0.3 & 0.5 & 0.5 & 0.4 & 0.8 \\
\hline Chemicals & 11.7 & 9.5 & 41.6 & 15.7 & 23.2 \\
\hline Manufactured goods classified chiefly by material & 715.3 & 741.6 & $1,016.0$ & $1,558.0$ & $2,772.1$ \\
\hline Machinery and transport equipment & 8.0 & 8.1 & 17.5 & 23.4 & 101.5 \\
\hline Miscellaneous manufactured articles & 18.5 & 10.8 & 22.2 & 59.3 & 10.0 \\
\hline \multirow[t]{2}{*}{ Other } & 24.0 & 117.4 & 270.4 & 34.2 & 138.0 \\
\hline & \multicolumn{5}{|c|}{ (In percent of total) } \\
\hline Exports, fob & 100.0 & 100.0 & 100.0 & 100.0 & 100.0 \\
\hline Food and live animals & 7.4 & 6.9 & 8.2 & 7.8 & 3.6 \\
\hline Beverages and tobacco & 1.7 & 2.0 & 3.3 & 3.4 & 2.0 \\
\hline Crude materials, (excl fuels) & 7.7 & 8.4 & 12.9 & 11.7 & 14.1 \\
\hline Mineral fuels, lubricants and related materials & 0.8 & 1.3 & 1.5 & 0.6 & 0.6 \\
\hline Animal and vegetable oils, fats and waxes & 0.0 & 0.0 & 0.0 & 0.0 & 0.0 \\
\hline Chemicals & 1.2 & 0.9 & 2.3 & 0.7 & 0.6 \\
\hline Manufactured goods classified chiefly by material & 75.8 & 68.0 & 55.0 & 70.5 & 72.6 \\
\hline Machinery and transport equipment & 0.8 & 0.7 & 0.9 & 1.1 & 2.7 \\
\hline Miscellaneous manufactured articles & 2.0 & 1.0 & 1.2 & 2.7 & 0.3 \\
\hline Other & 2.5 & 10.8 & 14.6 & 1.5 & 3.6 \\
\hline
\end{tabular}

Source: Central Statistics Office. 
Table 25. Zambia: Merchandise Imports by SITC

\begin{tabular}{|c|c|c|c|c|c|}
\hline & 2002 & 2003 & 2004 & 2005 & 2006 \\
\hline & \multicolumn{5}{|c|}{ (In million of U.S. dollars) } \\
\hline Imports, cif & $\ldots$ & $1,572.7$ & $2,149.6$ & $2,576.6$ & $3,022.4$ \\
\hline Food and live animals & $\ldots$ & 160.7 & 98.2 & 113.1 & 175.1 \\
\hline Beverages and tobacco & $\ldots$ & 2.1 & 2.3 & 3.2 & 10.3 \\
\hline Crude materials, (excl fuels) & $\ldots$ & 59.0 & 68.9 & 89.6 & 83.1 \\
\hline Mineral fuels, lubricants and related materials & $\ldots$ & 128.8 & 244.1 & 279.1 & 453.2 \\
\hline Animal and vegetable oils, fats and waxes & $\ldots$ & 33.2 & 41.2 & 41.7 & 48.9 \\
\hline Chemicals & $\ldots$ & 291.5 & 345.3 & 457.5 & 446.1 \\
\hline Manufactured goods classified chiefly by material & $\ldots$ & 249.7 & 314.2 & 405.8 & 429.1 \\
\hline Machinery and transport equipment & $\ldots$ & 504.4 & 670.6 & 814.2 & $1,207.2$ \\
\hline Miscellaneous manufactured articles & $\ldots$ & 143.0 & 364.8 & 372.4 & 169.3 \\
\hline \multirow[t]{2}{*}{ Other } & $\ldots$ & 0.1 & 0.1 & 0.0 & 0.1 \\
\hline & \multicolumn{5}{|c|}{ (In percent of total) } \\
\hline Imports, cif & $\ldots$ & 100.0 & 100.0 & 100.0 & 100.0 \\
\hline Food and live animals & $\ldots$ & 10.2 & 4.6 & 4.4 & 5.8 \\
\hline Beverages and tobacco & $\ldots$ & 0.1 & 0.1 & 0.1 & 0.3 \\
\hline Crude materials, (excl fuels) & $\ldots$ & 3.8 & 3.2 & 3.5 & 2.7 \\
\hline Mineral fuels, lubricants and related materials & $\ldots$ & 8.2 & 11.4 & 10.8 & 15.0 \\
\hline Animal and vegetable oils, fats and waxes & $\ldots$ & 2.1 & 1.9 & 1.6 & 1.6 \\
\hline Chemicals & $\ldots$ & 18.5 & 16.1 & 17.8 & 14.8 \\
\hline Manufactured goods classified chiefly by material & $\ldots$ & 15.9 & 14.6 & 15.7 & 14.2 \\
\hline Machinery and transport equipment & $\ldots$ & 32.1 & 31.2 & 31.6 & 39.9 \\
\hline Miscellaneous manufactured articles & $\ldots$ & 9.1 & 17.0 & 14.5 & 5.6 \\
\hline Other & $\ldots$ & 0.0 & 0.0 & 0.0 & 0.0 \\
\hline
\end{tabular}

Source: Central Statistics Office. 
Table 26. Zambia: Imports by Country (In millions of U.S. dollars)

\begin{tabular}{|c|c|c|c|c|c|}
\hline & 2002 & 2003 & 2004 & 2005 & 2006 \\
\hline Total imports, cif. & $\ldots$ & $1,572.7$ & $2,149.6$ & $2,576.6$ & $3,022.4$ \\
\hline European & $\ldots$ & 208.8 & 476.9 & 518.4 & 289.7 \\
\hline Belgium & $\ldots$ & 6.6 & 8.0 & 7.7 & 14.1 \\
\hline Finland & $\ldots$ & 27.0 & 22.7 & 12.1 & 12.9 \\
\hline France & $\ldots$ & 30.2 & 72.2 & 93.6 & 22.1 \\
\hline Germany & $\ldots$ & 24.0 & 22.1 & 35.8 & 44.1 \\
\hline Italy & $\ldots$ & 5.6 & 4.8 & 9.8 & 16.4 \\
\hline Netherlands & $\ldots$ & 10.9 & 11.2 & 30.6 & 45.5 \\
\hline Switzerland & $\ldots$ & 11.2 & 18.1 & 16.1 & 23.2 \\
\hline United Kingdom & $\ldots$ & 93.3 & 317.8 & 312.7 & 111.4 \\
\hline Other European Countries & $\ldots$ & 25.3 & 39.3 & 67.2 & 213.9 \\
\hline SADC members & $\ldots$ & $1,040.9$ & $1,195.8$ & $1,479.0$ & $1,750.5$ \\
\hline Democratic Republic Of Congo & $\ldots$ & 12.1 & 14.1 & 23.3 & 30.5 \\
\hline Angola & $\ldots$ & 0.0 & 0.1 & 0.0 & 0.0 \\
\hline Botswana & $\ldots$ & 6.5 & 9.4 & 11.9 & 18.5 \\
\hline Lesotho & $\ldots$ & 0.0 & 0.0 & 0.0 & 0.0 \\
\hline Madagascar & $\ldots$ & 0.0 & 0.0 & 0.0 & 0.0 \\
\hline Malawi & $\ldots$ & 9.4 & 7.8 & 14.0 & 12.9 \\
\hline Mauritius & $\ldots$ & 2.4 & 2.8 & 2.0 & 2.4 \\
\hline Mozambique & $\ldots$ & 10.6 & 2.3 & 10.5 & 16.4 \\
\hline Namibia & $\ldots$ & 3.6 & 5.7 & 6.4 & 8.3 \\
\hline South Africa (Republic Of) & $\ldots$ & 765.4 & 993.5 & $1,228.5$ & $1,430.6$ \\
\hline Swaziland & $\ldots$ & 4.3 & 5.4 & 3.7 & 5.2 \\
\hline Tanzania & $\ldots$ & 23.6 & 30.1 & 68.5 & 54.1 \\
\hline Zimbabwe & $\ldots$ & 202.9 & 124.6 & 110.2 & 171.5 \\
\hline Other Trading Partners & $\ldots$ & 323.0 & 476.9 & 579.1 & 982.2 \\
\hline China & $\ldots$ & 44.3 & 46.1 & 83.8 & 81.4 \\
\hline Egypt & $\ldots$ & 2.0 & 3.8 & 6.3 & 6.6 \\
\hline India & $\ldots$ & 35.3 & 50.6 & 80.6 & 85.2 \\
\hline Japan & $\ldots$ & 23.3 & 28.8 & 41.9 & 47.6 \\
\hline Kenya & $\ldots$ & 24.0 & 34.1 & 38.0 & 55.3 \\
\hline Korea, Republic Of & $\ldots$ & 8.2 & 12.4 & 15.3 & 8.5 \\
\hline Malaysia & $\ldots$ & 2.8 & 1.9 & 1.9 & 3.2 \\
\hline Pakistan & $\ldots$ & 2.5 & 2.5 & 2.0 & 1.4 \\
\hline Republic Of Thailand & $\ldots$ & 1.5 & 3.0 & 5.4 & 5.2 \\
\hline Saudi Arabia & $\ldots$ & 1.6 & 4.8 & 0.3 & 1.7 \\
\hline Taiwan, Province Of China & $\ldots$ & 6.9 & 10.1 & 10.2 & 11.0 \\
\hline United Arab Emirates & $\ldots$ & 66.9 & 145.1 & 96.1 & 313.0 \\
\hline United States Of America & $\ldots$ & 32.3 & 34.9 & 41.9 & 57.2 \\
\hline Other & $\ldots$ & 71.6 & 98.7 & 155.5 & 304.8 \\
\hline \multicolumn{6}{|c|}{ (In percent of total) } \\
\hline European countries & $\ldots$ & 13.3 & 22.2 & 20.1 & 9.6 \\
\hline SADC members & $\ldots$ & 66.2 & 55.6 & 57.4 & 57.9 \\
\hline Others & $\ldots$ & 20.5 & 22.2 & 22.5 & 32.5 \\
\hline
\end{tabular}

Source: Central Statistics Office. 
Table 27. Zambia: Exports by Country

(In millions of U.S. dollars)

\begin{tabular}{|c|c|c|c|c|c|}
\hline & 2002 & 2003 & 2004 & 2005 & 2006 \\
\hline Total exports, fob & 944.3 & $1,090.4$ & $1,846.9$ & $2,210.4$ & $3,819.2$ \\
\hline European & 513.6 & 414.7 & 663.9 & 975.1 & $1,675.1$ \\
\hline Belgium & 17.5 & 28.0 & 36.6 & 31.4 & 9.0 \\
\hline Finland & 5.7 & 11.9 & 11.4 & 0.0 & 8.3 \\
\hline France & 2.9 & 5.1 & 16.7 & 13.1 & 16.7 \\
\hline Germany & 9.0 & 7.4 & 10.8 & 14.6 & 11.1 \\
\hline Italy & 2.5 & 1.6 & 3.1 & 7.9 & 3.4 \\
\hline Netherlands & 20.9 & 27.5 & 47.8 & 46.0 & 44.3 \\
\hline Switzerland & 57.4 & 76.8 & 255.5 & 754.1 & $1,462.6$ \\
\hline United Kingdom & 393.6 & 253.5 & 275.1 & 74.5 & 99.4 \\
\hline Other European Countries & 4.0 & 2.9 & 6.8 & 33.5 & 20.1 \\
\hline SADC members & 348.2 & 430.3 & 766.7 & 510.0 & 684.3 \\
\hline Democratic Republic Of Congo & 38.9 & 41.6 & 106.0 & 102.0 & 128.4 \\
\hline Angola & 0.4 & 1.1 & 0.4 & 0.6 & 0.4 \\
\hline Botswana & 2.7 & 3.6 & 2.9 & 40.4 & 6.0 \\
\hline Lesotho & 0.1 & 0.1 & 2.5 & 0.7 & 4.5 \\
\hline Madagascar & 0.0 & 0.0 & 0.0 & 0.0 & 0.0 \\
\hline Malawi & 15.4 & 24.4 & 48.1 & 70.8 & 57.6 \\
\hline Mauritius & 6.2 & 3.9 & 3.8 & 1.3 & 0.7 \\
\hline Mozambique & 0.1 & 1.2 & 1.5 & 0.7 & 1.3 \\
\hline Namibia & 1.8 & 1.3 & 4.3 & 8.7 & 17.6 \\
\hline South Africa (Republic Of) & 211.8 & 211.2 & 386.4 & 195.9 & 401.4 \\
\hline Swaziland & 0.6 & 0.2 & 0.5 & 0.9 & 0.9 \\
\hline Tanzania & 54.1 & 122.9 & 120.9 & 11.5 & 13.8 \\
\hline Zimbabwe & 16.0 & 18.9 & 89.4 & 76.4 & 51.7 \\
\hline Other Trading Partners & 82.5 & 245.4 & 416.3 & 725.3 & $1,459.8$ \\
\hline China & 3.3 & 16.5 & 33.9 & 192.1 & 257.7 \\
\hline Egypt & 0.9 & 1.0 & 0.7 & 79.5 & 151.1 \\
\hline India & 29.0 & 34.2 & 16.8 & 32.8 & 133.3 \\
\hline Japan & 7.7 & 16.2 & 7.0 & 29.8 & 3.7 \\
\hline Kenya & 4.3 & 5.8 & 9.1 & 13.8 & 30.7 \\
\hline Korea, Republic Of & 0.3 & 0.1 & 0.0 & 65.1 & 44.4 \\
\hline Malaysia & 0.0 & 0.0 & 0.0 & 52.7 & 77.2 \\
\hline Pakistan & 0.0 & 0.0 & 0.0 & 24.6 & 49.2 \\
\hline Republic Of Thailand & 1.8 & 6.2 & 1.1 & 98.9 & 284.7 \\
\hline Saudi Arabia & 0.1 & 0.0 & 0.0 & 60.2 & 84.3 \\
\hline Taiwan, Province Of China & 0.0 & 2.1 & 44.7 & 2.9 & 71.4 \\
\hline United States Of America & 9.7 & 6.9 & 17.7 & 14.2 & 2.2 \\
\hline Other & 25.3 & 156.4 & 285.2 & 58.8 & 269.9 \\
\hline & \multicolumn{5}{|c|}{ (In percent of total) } \\
\hline European countries & 54.4 & 38.0 & 35.9 & 44.1 & 43.9 \\
\hline SADC members & 36.9 & 39.5 & 41.5 & 23.1 & 17.9 \\
\hline Others & 8.7 & 22.5 & 22.5 & 32.8 & 38.2 \\
\hline
\end{tabular}

Source: Central Statistics Office. 
Table 28. Zambia: Scheduled External Debt Service Payments

\begin{tabular}{|c|c|c|c|c|c|}
\hline & 2002 & 2003 & 2004 & 2005 & 2006 \\
\hline & \multicolumn{5}{|c|}{ (In millions of U.S. dollars) } \\
\hline Scheduled debt service & 588 & 612 & 709 & 2380 & 2469 \\
\hline Interest & 132 & 126 & 121 & 110 & 17 \\
\hline Of which: Fund charges/interest & 3 & 6 & 2 & 4 & 0 \\
\hline Amortization & 456 & 486 & 588 & 2270 & 2452 \\
\hline \multirow[t]{2}{*}{ Of which: Fund repurchases } & 222 & 244 & 457 & 252 & 582 \\
\hline & \multicolumn{5}{|c|}{ (In percent of exports of goods and nonfactor services) } \\
\hline Scheduled debt service & 54.4 & 48.7 & 34.1 & 95.9 & 59.9 \\
\hline Interest & 12.2 & 10.0 & 5.8 & 4.4 & 0.4 \\
\hline Of which: Fund charges/interest & 0.3 & 0.5 & 0.1 & 0.2 & 0.0 \\
\hline Amortization & 42.2 & 38.7 & 28.3 & 91.4 & 59.5 \\
\hline Of which: Fund repurchases & 20.6 & 19.4 & 22.0 & 10.2 & 14.1 \\
\hline \multicolumn{6}{|l|}{ Memorandum item: } \\
\hline \multicolumn{6}{|l|}{ Exports of goods and services } \\
\hline (in millions of U.S. dollars) & 1,080 & 1,255 & 2,079 & 2,483 & 4,122 \\
\hline
\end{tabular}

Sources: Bank of Zambia; and staff estimates. 
Table 29. Zambia: Central Government External Debt 1/

\begin{tabular}{|c|c|c|c|c|c|}
\hline & 2002 & 2003 & 2004 & 2005 & 2006 \\
\hline & \multicolumn{5}{|c|}{ (In millions of U.S. dollars unless otherwise indicated) } \\
\hline Gross external debt & 6,746 & 6,769 & 6,894 & 6,254 & 961 \\
\hline In percent of GDP & 178.7 & 156.5 & 126.7 & 86.0 & 8.8 \\
\hline Medium- and long-term debt 2 & 6,746 & 6,769 & 6,894 & 6254.3 & 960.6 \\
\hline Multilateral & 3,499 & 3,995 & 4,211 & 3570.3 & 600.1 \\
\hline IMF & 1,000 & 848 & 883 & 591.9 & 41.5 \\
\hline Other & 2,499 & 3,147 & 3,328 & 2978.4 & 558.6 \\
\hline Bilateral official & 2,615 & 2,679 & 2,602 & 2609.1 & 298.9 \\
\hline Paris Club & 2,343 & 2,453 & 2,379 & 2388.0 & 204.2 \\
\hline Other & 272 & 226 & 223 & 221.1 & 94.7 \\
\hline \multirow[t]{2}{*}{ Suppliers and other $3 /$} & 632 & 95 & 81 & 74.9 & 61.6 \\
\hline & \multicolumn{5}{|c|}{ (In percent of exports of goods and services) } \\
\hline Gross external debt & 624.5 & 539.2 & 331.7 & 251.9 & 23.3 \\
\hline Medium- and long-term debt 2 & 624.5 & 539.2 & 331.7 & 251.9 & 23.3 \\
\hline Multilateral & 323.9 & 318.2 & 202.6 & 143.8 & 14.6 \\
\hline IMF & 92.6 & 67.5 & 42.5 & 23.8 & 1.0 \\
\hline Other & 231.3 & 250.7 & 160.1 & 120.0 & 13.6 \\
\hline Bilateral official & 242.0 & 213.4 & 125.2 & 105.1 & 7.3 \\
\hline Paris Club & 216.9 & 195.4 & 114.4 & 96.2 & 5.0 \\
\hline Other & 25.2 & 18.0 & 10.7 & 8.9 & 2.3 \\
\hline Suppliers and other $3 /$ & 58.5 & 7.6 & 3.9 & 3.0 & 1.5 \\
\hline \multicolumn{6}{|l|}{ Memorandum item: } \\
\hline $\begin{array}{l}\text { Exports of goods and services } \\
\text { (in millions of U.S. dollars) }\end{array}$ & 1,080 & 1,255 & 2,079 & 2,483 & 4,122 \\
\hline
\end{tabular}

Sources: Bank of Zambia; and staff estimates.

1/ Not including debt relief under the HIPC Initiative.

2/ Including arrears.

3/ Excludes "dormant" commercial debt not tendered in 1994 buyback, which the authorities estimate to amount to US\$85 million. 
Table 30. Zambia: Nominal, Nominal Effective, and Real Effective

Exchange Rates

(Index, 1995=100, unless otherwise indicated)

\begin{tabular}{|c|c|c|c|c|}
\hline & $\begin{array}{r}\text { Official } \\
\text { Kwacha-U.S. Dollar } \\
\text { Exchange Rate }\end{array}$ & $\begin{array}{r}\text { Official } \\
\text { U.S. Dollar-Kwacha } \\
\text { Exchange Rate }\end{array}$ & $\begin{array}{r}\text { Nominal } \\
\text { Effective } \\
\text { Exchange Rate }\end{array}$ & $\begin{array}{r}\text { Real } \\
\text { Effective } \\
\text { Exchange Rate }\end{array}$ \\
\hline 1995 Q1 & 759.3 & 0.001317 & 116.5 & 105.5 \\
\hline Q2 & 849.8 & 0.001177 & 99.8 & 93.4 \\
\hline Q3 & 940.3 & 0.001063 & 91.4 & 92.4 \\
\hline Q4 & 943.7 & 0.001060 & 92.3 & 108.7 \\
\hline 1996 Q1 & $1,053.2$ & 0.000949 & 84.7 & 106.4 \\
\hline Q2 & $1,238.3$ & 0.000808 & 73.1 & 99.7 \\
\hline Q3 & $1,264.6$ & 0.000791 & 70.9 & 103.1 \\
\hline Q4 & $1,273.8$ & 0.000785 & 71.0 & 109.4 \\
\hline 1997 Q1 & $1,289.5$ & 0.000775 & 73.4 & 119.8 \\
\hline Q2 & $1,295.9$ & 0.000772 & 73.9 & 122.5 \\
\hline Q3 & $1,315.5$ & 0.000760 & 74.8 & 128.2 \\
\hline Q4 & $1,357.4$ & 0.000737 & 72.7 & 131.0 \\
\hline 1998 Q1 & $1,544.2$ & 0.000648 & 66.5 & 124.6 \\
\hline Q2 & $1,825.9$ & 0.000548 & 56.4 & 113.4 \\
\hline Q3 & $1,941.2$ & 0.000515 & 53.6 & 114.2 \\
\hline Q4 & $2,136.0$ & 0.000468 & 47.2 & 106.1 \\
\hline 1999 Q1 & $2,288.1$ & 0.000437 & 44.9 & 106.9 \\
\hline Q2 & $2,377.9$ & 0.000421 & 44.5 & 111.0 \\
\hline Q3 & $2,403.6$ & 0.000416 & 43.9 & 115.6 \\
\hline Q4 & $2,482.5$ & 0.000403 & 42.3 & 114.3 \\
\hline 2000 Q1 & $2,714.4$ & 0.000368 & 39.6 & 111.7 \\
\hline Q2 & $2,866.4$ & 0.000349 & 38.8 & 117.0 \\
\hline Q3 & $3,177.6$ & 0.000315 & 36.0 & 116.0 \\
\hline Q4 & $3,685.1$ & 0.000271 & 32.3 & 108.6 \\
\hline 2001 Q1 & $3,658.9$ & 0.000273 & 34.4 & 120.9 \\
\hline Q2 & $3,344.0$ & 0.000299 & 36.4 & 129.4 \\
\hline Q2 & $3,652.6$ & 0.000274 & 32.8 & 119.9 \\
\hline Q2 & $3,788.3$ & 0.000264 & 31.7 & 121.4 \\
\hline 2002 Q1 & $3,894.5$ & 0.000257 & 31.5 & 126.4 \\
\hline Q2 & $4,130.4$ & 0.000242 & 28.7 & 118.8 \\
\hline Q3 & $4,503.7$ & 0.000222 & 25.1 & 108.8 \\
\hline Q4 & $4,699.0$ & 0.000213 & 23.9 & 109.1 \\
\hline 2003 Q1 & $4,652.7$ & 0.000215 & 23.8 & 110.9 \\
\hline Q2 & $4,845.0$ & 0.000206 & 23.3 & 111.8 \\
\hline Q3 & $4,740.4$ & 0.000211 & 23.8 & 116.8 \\
\hline Q4 & $4,697.9$ & 0.000213 & 22.9 & 115.5 \\
\hline 2004 Q1 & $4,750.7$ & 0.000210 & 22.7 & 116.3 \\
\hline Q2 & $4,774.1$ & 0.000209 & 23.3 & 123.6 \\
\hline Q3 & $4,808.9$ & 0.000208 & 23.0 & 126.2 \\
\hline Q4 & $4,781.1$ & 0.000209 & 22.4 & 126.3 \\
\hline 2005 Q1 & $4,677.8$ & 0.000214 & 22.2 & 128.5 \\
\hline Q2 & $4,676.2$ & 0.000214 & 23.3 & 140.4 \\
\hline Q3 & $4,503.3$ & 0.000222 & 25.5 & 156.6 \\
\hline Q4 & $3,929.7$ & 0.000254 & 41.6 & 198.5 \\
\hline 2006 Q1 & $3,315.8$ & 0.000302 & 48.3 & 222.9 \\
\hline Q2 & $3,286.3$ & 0.000304 & 49.1 & 224.3 \\
\hline Q3 & $3,825.7$ & 0.000261 & 44.7 & 203.2 \\
\hline Q4 & $3,984.5$ & 0.000251 & 43.5 & 196.0 \\
\hline
\end{tabular}

Source: IMF ( Information Notice System). 


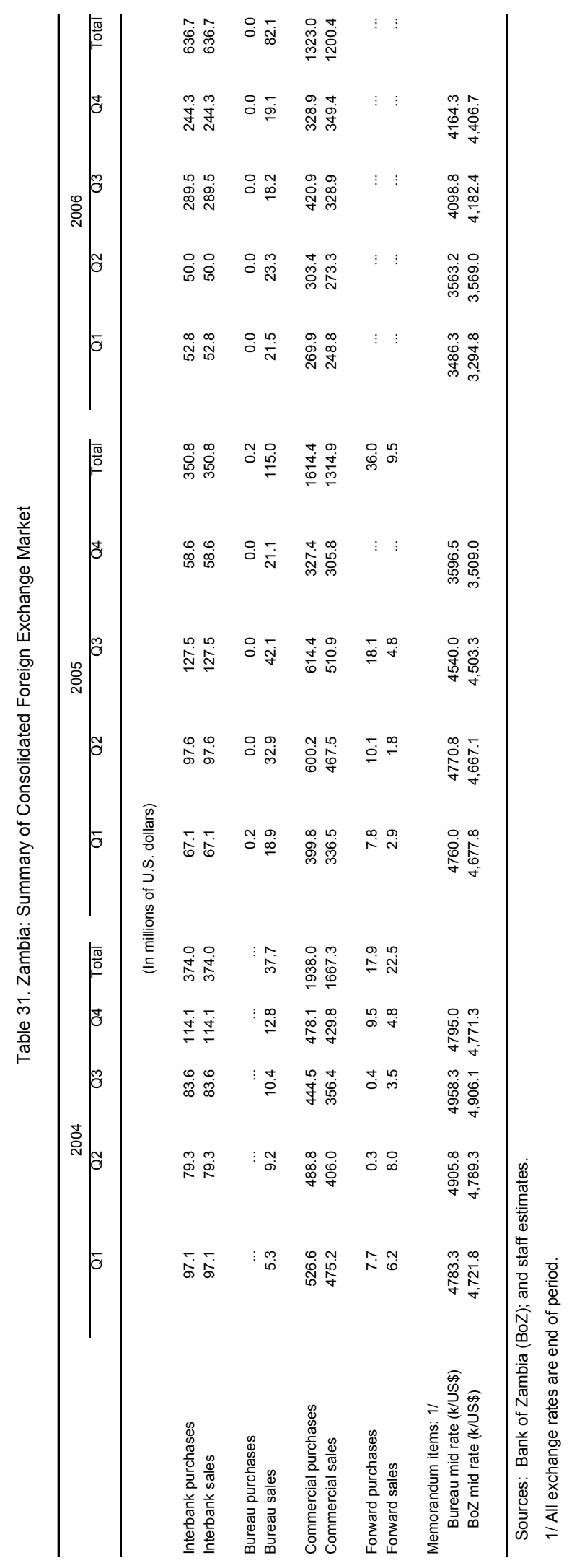


Annex I. Zambia: Millennium Development Goals

Goal 1: Eradicate extreme poverty and hunger

Income share held by lowest $20 \%$

Malnutrition prevalence, weight for age ( $\%$ of children under 5$)$

Poverty gap at $\$ 1$ a day (PPP) (\%)

Poverty headcount ratio at $\$ 1$ a day (PPP) (\% of population)

Poverty headcount ratio at national poverty line ( $\%$ of population)

Prevalence of undernourishment (\% of population)

Goal 2: Achieve universal primary education

Literacy rate, youth total (\% of people ages 15-24)

Persistence to grade 5, total ( $\%$ of cohort)

Primary completion rate, total ( $\%$ of relevant age group)

School enrollment, primary ( $\%$ net)

Goal 3: Promote gender equality and empower women

Proportion of seats held by women in national parliament (\%)

Ratio of girls to boys in primary and secondary education (\%)

Ratio of young literate females to males (\% ages 15-24)

Share of women employed in the nonagricultural sector (\% of total nonagricultural employment)

Goal 4: Reduce child mortality

Immunization, measles ( $\%$ of children ages $12-23$ months)

Mortality rate, infant (per 1,000 live births)

Mortality rate, under-5 (per 1,000)

Goal 5: Improve maternal health

Births attended by skilled health staff ( $\%$ of total)

Maternal mortality ratio (modeled estimate, per 100,000 live births)

Goal 6: Combat HIV/AIDS, malaria, and other diseases

Contraceptive prevalence ( $\%$ of women ages $15-49$ )

Incidence of tuberculosis (per 100,000 people)

Prevalence of HIV, female (\% ages 15-24)

Prevalence of HIV, total ( $\%$ of population ages 15-49)

Tuberculosis cases detected under DOTS (\%)

Goal 7: Ensure environmental sustainability

$\mathrm{CO} 2$ emissions (metric tons per capita)

Forest area (\% of land area)

GDP per unit of energy use (constant 2000 PPP \$ per kg of oil equivalent)

Improved sanitation facilities ( $\%$ of population with access)

Improved water source ( $\%$ of population with access)

Nationally protected areas ( $\%$ of total land area)

Goal 8: Develop a global partnership for development

Aid per capita (current US\$)

Debt service (PPG and IMF only, \% of exports of G\&S, excl. workers' remittances)

Fixed line and mobile phone subscribers (per 1,000 people)

Internet users (per 1,000 people)

Personal computers (per 1,000 people)

Total debt service ( $\%$ of exports of goods, services and income)

Unemployment, youth female (\% of female labor force ages 15-24)

Unemployment, youth male ( $\%$ of male labor force ages $15-24$ )

Unemployment, youth total (\% of total labor force ages 15-24)

Other

Fertility rate, total (births per woman)

GNI per capita, Atlas method (current US\$)

GNI, Atlas method (current US\$) (billions)

Gross capital formation ( $\%$ of GDP)

Life expectancy at birth, total (years)

Literacy rate, adult total (\% of people ages 15 and above)

Population, total (millions)

Trade (\% of GDP)

$\begin{array}{llll}1.3 & 4.2 & 3.4 & 3.6\end{array}$

$\begin{array}{llll}25.2 & 23.5 & 25.0 & 23.0\end{array}$

$\begin{array}{llll}38.9 & 37.8 & 34.0 & 32.6\end{array}$

$\begin{array}{llll}64.6 & 72.6 & 65.7 & 63.8\end{array}$

$\begin{array}{rrrr}. . & . . & 72.9 & 68.0\end{array}$

$\begin{array}{llll}48 & 48 & . . & 46\end{array}$

$\begin{array}{llll}81 & . . & . . & 69\end{array}$

$\begin{array}{llll}. . & . . & 98 & \\ . & . .\end{array}$

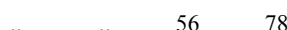

$\begin{array}{llll}. . & . . & 63 & 89\end{array}$

$\begin{array}{llll}7 & 10 & 10 & 13\end{array}$

$\begin{array}{llll}. . & . . & 90 & 92\end{array}$

$\begin{array}{llll}88 & . . & . . & 91\end{array}$

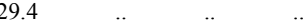

$\begin{array}{lrrr}90 & 86 & 85 & 84\end{array}$

$\begin{array}{llll}101 & 102 & 102 & 102\end{array}$

$\begin{array}{llll}180 & 182 & 182 & 182\end{array}$

$\begin{array}{llll}51 & 47 & 47 \quad . .\end{array}$

.. $\quad . . \quad 750 \quad$..

$\begin{array}{llll}15 & 26 & 54 & \end{array}$

$\begin{array}{llll}297 & 534 & 605 & 600\end{array}$

$\begin{array}{llll}. . & . . & . . & 12.7\end{array}$

$\begin{array}{llll}. . & . . & 15.6 & 17.0\end{array}$

$\begin{array}{llll}. . & . . & 40 & 52\end{array}$

$\begin{array}{llll}0.3 & 0.2 & 0.2 & 0.2\end{array}$

$\begin{array}{llll}66 & . . & 60 & 57\end{array}$

$\begin{array}{llll}1.5 & 1.3 & 1.3 & 1.5\end{array}$

$\begin{array}{rrrr}44 & . . & & \\ 44 & . . & 55\end{array}$

50

$\begin{array}{llll}. . & . . & . . & 42.0\end{array}$

$\begin{array}{llll}57 & 212 & 74 & 81\end{array}$

$\begin{array}{llll}14.5 & 18.3 & 15.7 & 6.7\end{array}$

$\begin{array}{llrr}8 & 8 & 17 & 89\end{array}$

$\begin{array}{llll}0 & 0 & 2 & 20\end{array}$

$\begin{array}{llll}. . & . . & 7 & 10\end{array}$

$\begin{array}{rrrr}14.7 & 19.4 & 20.2 & . . \\ 21.1 & . . & . . & . .\end{array}$

$\begin{array}{llll}21.7 & . . & . . & . .\end{array}$

$\begin{array}{lllll}20.9 & . . & . . & . .\end{array}$

$\begin{array}{llll}6.5 & 6.2 & 5.9 & 5.4\end{array}$

$\begin{array}{llll}420 & 320 & 290 & 500\end{array}$

$\begin{array}{llll}3.5 & 3.1 & 3.1 & 5.8\end{array}$

$\begin{array}{rrrr}17.3 & 15.9 & 18.7 & 25.8\end{array}$

$\begin{array}{rrrr}46 & 41 & 38 & 38\end{array}$

$\begin{array}{llll}68 & . . & . . & 68\end{array}$

$\begin{array}{llll}8.4 & 9.6 & 10.7 & 11.7\end{array}$

$\begin{array}{llll}72.5 & 75.8 & 52.5 & 41.6\end{array}$

Source: World Development Indicators database, April 2007. 
Annex II. Zambia: Government Shares in Public Enterprises

\begin{tabular}{lr}
\hline Entities & As of: \\
& Sept. 2007 \\
\hline
\end{tabular}

Energy sector

Indeni Petroleum Products $\quad 50$

$\begin{array}{lr}\text { Tazama Pipelines } & 60\end{array}$

$\begin{array}{lr}\text { Zambia Electricity Supply Corporation } & 100\end{array}$

Mining sector

Kagem Mining 20

Kariba Minerals $\quad 51$

$\begin{array}{lr}\text { Maamba Collieries } & 100\end{array}$

$\begin{array}{lr}\text { Nitrogen Chemicals of Zambia } & 100\end{array}$

Zambia Consolidated Copper Mining Investment Holding 87.6

$\begin{array}{lr}\text { AHC Mining Municipal Services } & 100\end{array}$

$\begin{array}{ll}\text { Chambishi Metals } & 10\end{array}$

Chibuluma Mines 15

$\begin{array}{ll}\text { Copperbelt Energy Corporation } & 20\end{array}$

Kansanshi mining $\quad 20$

Konkola Copper Mines $\quad 20.6$

Luanshya Copper Mines $\quad 15$

Mopani Copper Mines $\quad 10$

Ndola Lime Company $\quad 100$

NFC Africa Mining 15

Financial sector

$\begin{array}{ll}\text { Development Bank of Zambia } & 67\end{array}$

Indo-Zambia Bank Limited $\quad 50$

$\begin{array}{lr}\text { National Savings and Credit Bank } & 100\end{array}$

Zambia National Building Society $\quad 100$

Zambia National Commercial Bank $\quad 51$

Zambia State Insurance Company $\quad 100$

Other sectors

Contract Haulage

100

Engineering Services corporation $\quad 100$

$\begin{array}{ll}\text { Medical stores } & 100\end{array}$

MOFED (London) 100

MOFED (Tanzania) $\quad 100$

$\begin{array}{ll}\text { Mpongwe Development } & 0.05\end{array}$

Mpulungu Harbour Corporation $\quad 100$

Mupepetwe Engineering $\quad 100$

National Airports Corporation 100

$\begin{array}{lr}\text { National Housing Authority } & 100\end{array}$

$\begin{array}{ll}\text { Nganga Farms } & 23.8\end{array}$

Tanzania-Zambia Railways $\quad 50$

$\begin{array}{ll}\text { Times of Zambia } & 100\end{array}$

Zambia China Mulungushi Textiles of Zambia $\quad 40$

Zambia Daily Mail $\quad 100$

Zambia Education and Publishing House 100

$\begin{array}{lr}\text { Zambia Postal Corporation } & 100\end{array}$

Zambia Printing company 100

$\begin{array}{ll}\text { Zambia Railways } & 100\end{array}$

Zambia State Lotteries $\quad 100$

Zambia Telecommunication $\quad 100$

$\begin{array}{ll}\text { Zamcapital Enterprises } & 100\end{array}$

Sources: Ministry of Finance and National Planning (IDM). 


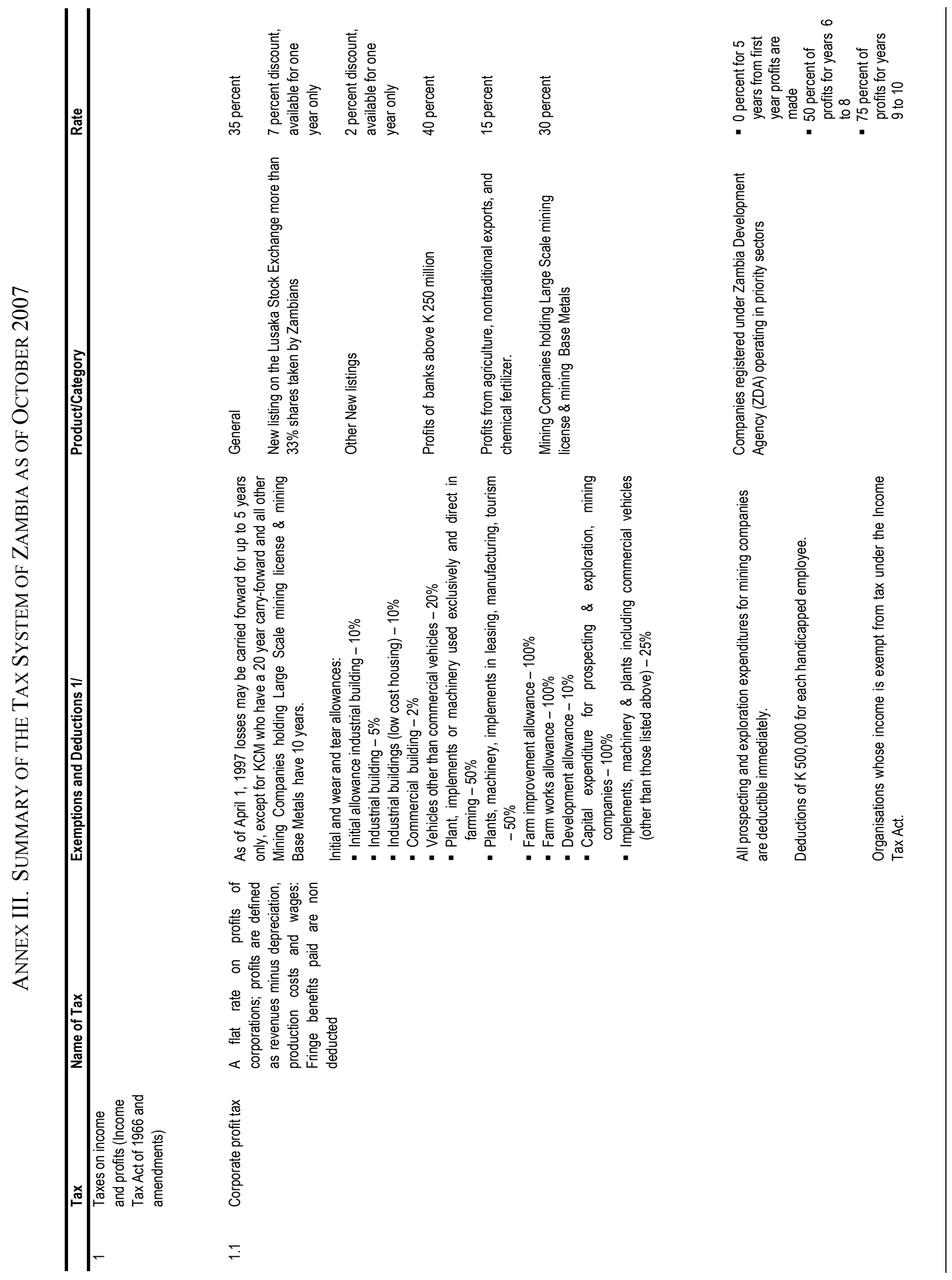



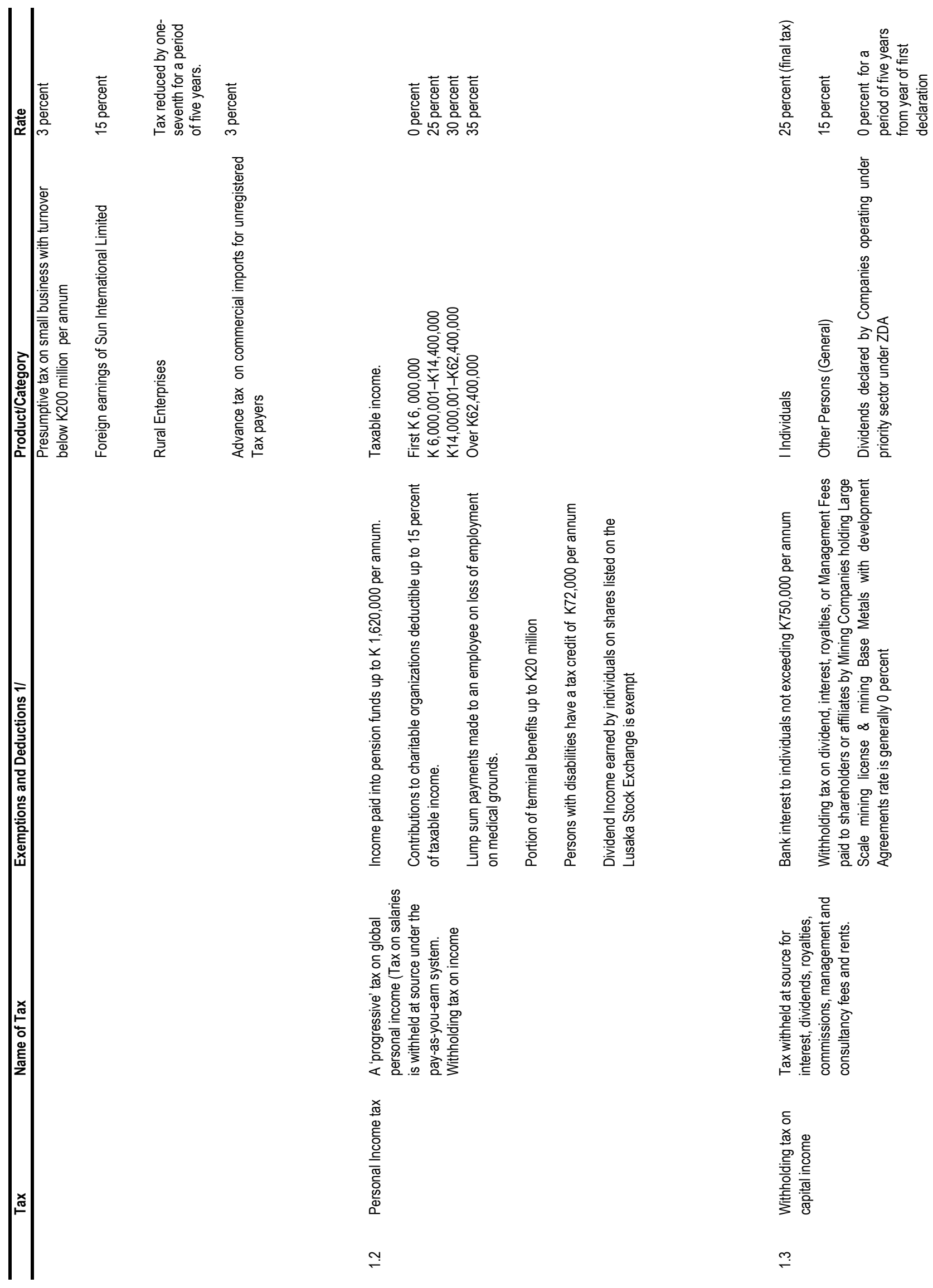

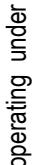

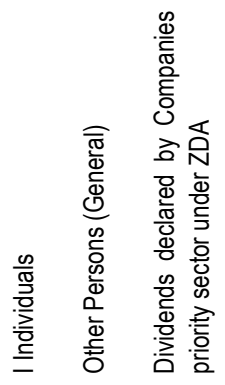

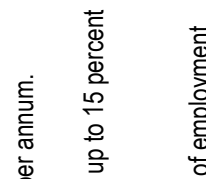

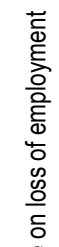

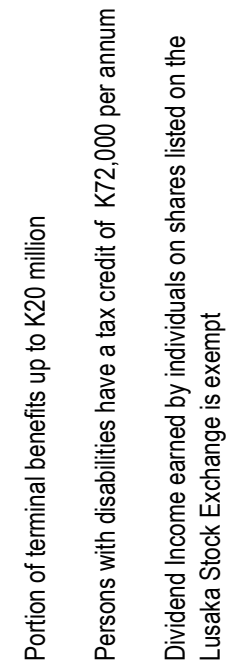

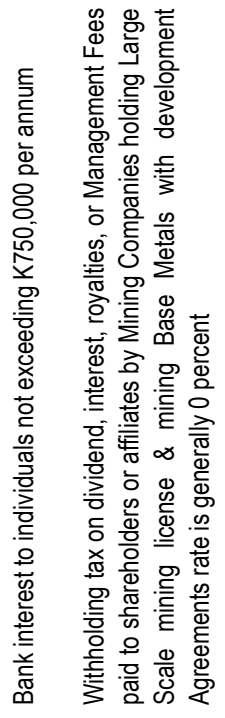
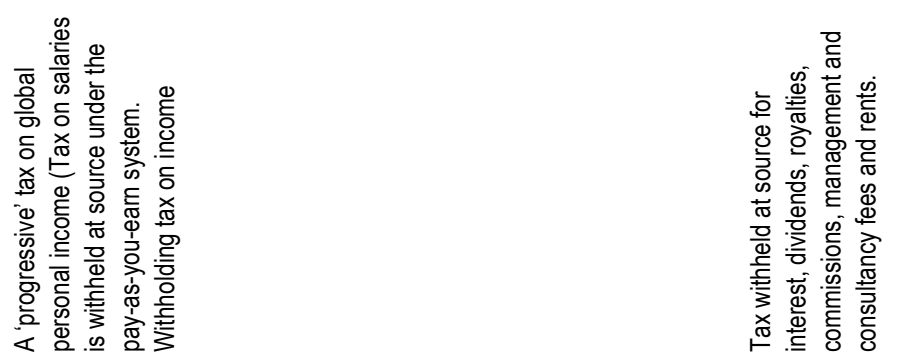

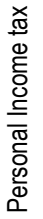

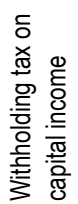




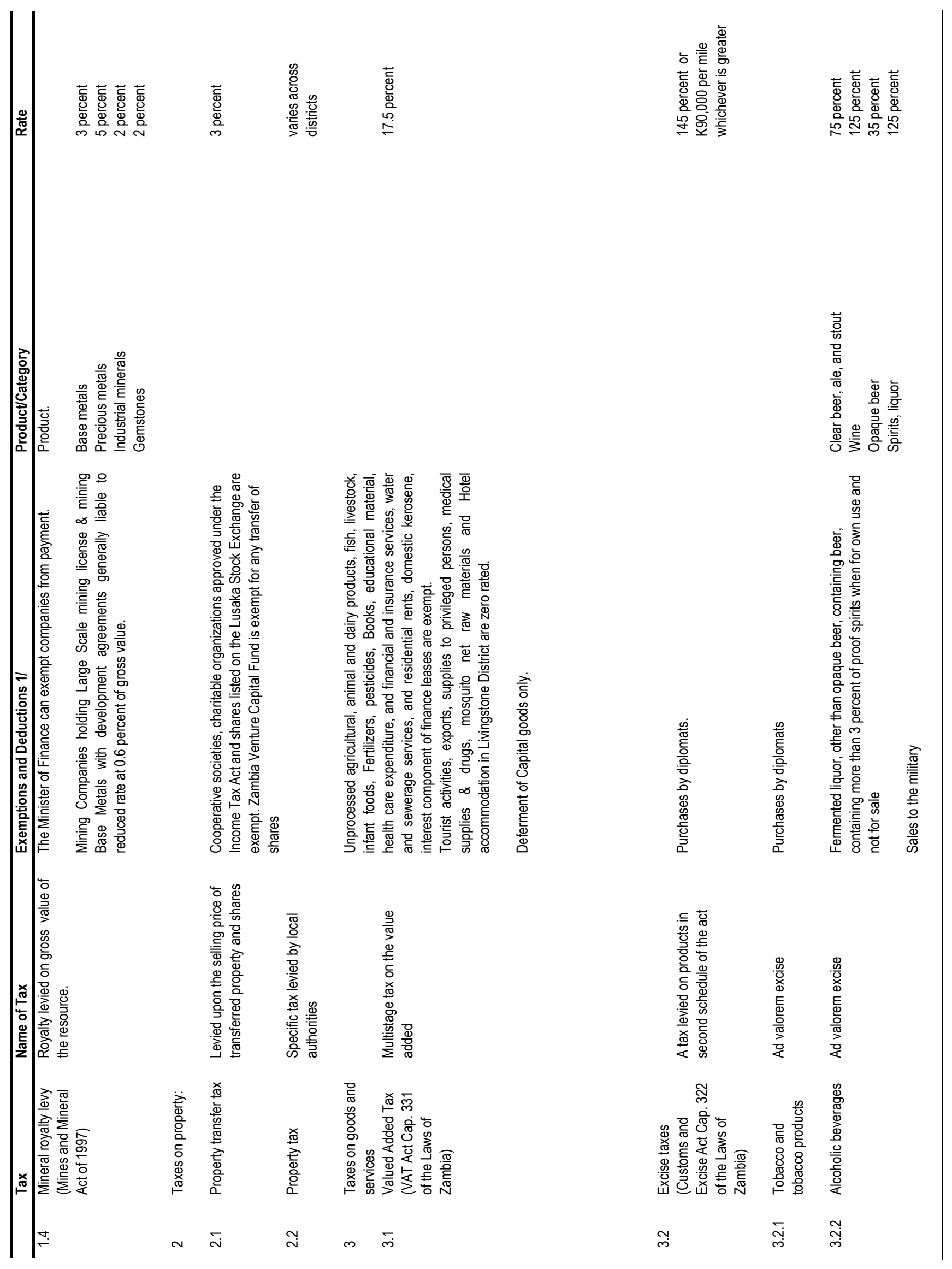




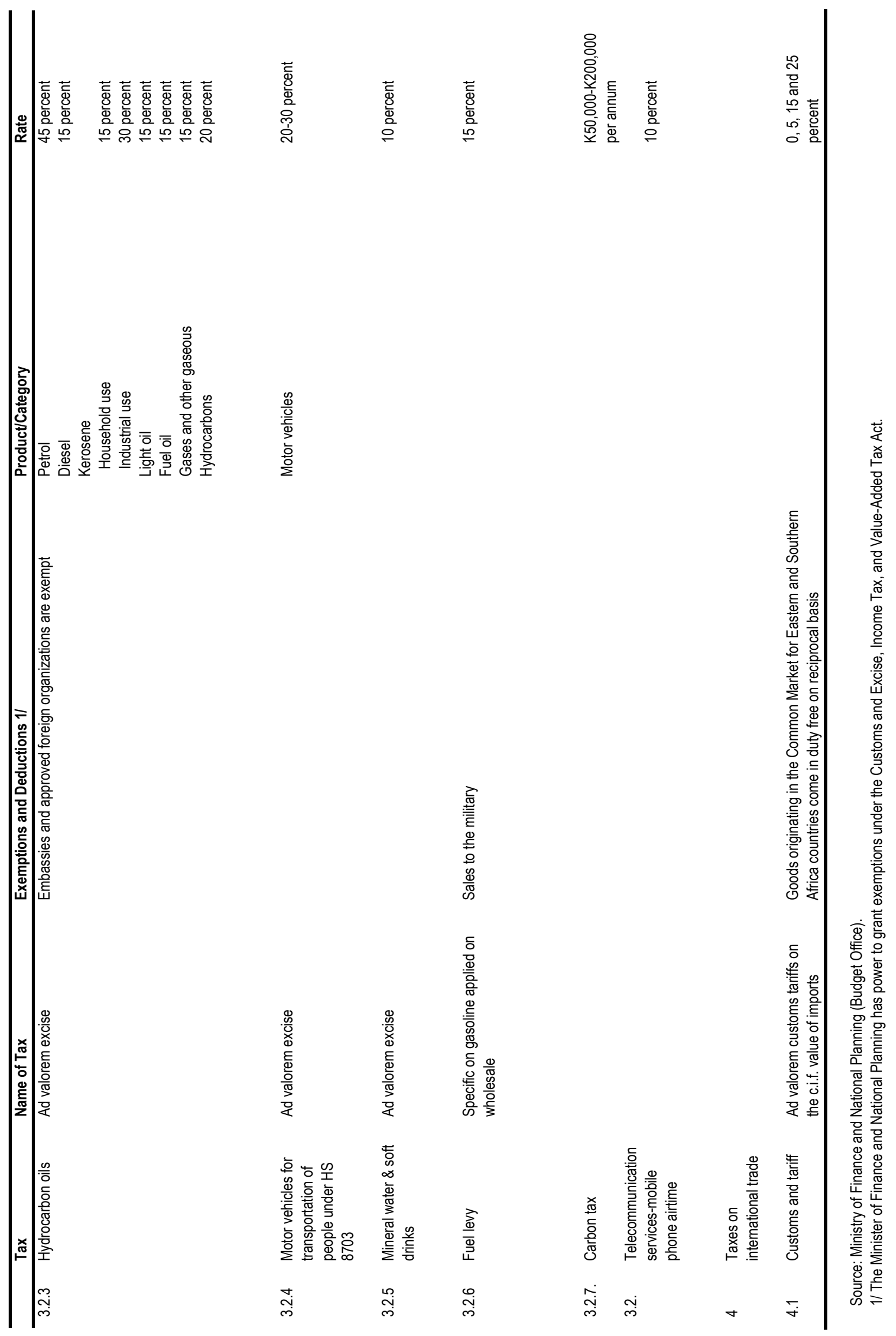

